\title{
Analysis of fluctuating force acting on two cylinders in different arrangements through Lattice Boltzmann Method
}

4

\author{
H. Jiao, G.X. Wu ${ }^{\dagger}$
}

Department of Mechanical Engineering, University College London, London WClE 7JE, UK

\section{Abstract}

The force components on two circular cylinders in tandem, side-by-side and staggered arrangements and in a uniform incoming flow are investigated systematically with Reynolds number being taken $\operatorname{Re}=200$. The numerical simulations are undertaken using the lattice Boltzmann method and the boundary condition is imposed though the immersed boundary method. At an intermediate centre-to-centre spacing which is based on the ratio of distance between cylinder centres and the cylinder diameter, the fluid force is periodic and dominated virtually by a single frequency component in the tandem arrangement and is random with a continuous frequency spectrum in the side-by-side arrangement. Here random means that the components of the force are not in the form of discrete frequencies, but are in the form of continuous spectrum. Detailed analysis has been undertaken to show this evolution process with the alignment angle $\alpha$, which is defined as the angle between the incoming flow direction and the line linking two cylinders. As $\alpha$ increases, the frequency component of the force on each individual cylinder changes from a single value to multiple ones, then to a large number of discrete ones and eventually to a broadband continuous spectrum. The results have important implications to vortex induced vibration and fatigue analysis of the cylinder, which has important applications, such as risers in offshore engineering.

Keywords: two cylinders in different arrangements; force components; lattice Boltzmann method; immersed boundary method

\section{Introduction}

†Email address: g.wu@ucl.ac.uk 
1 The flow past bluff bodies has been a subject of extensive interest due to its high complex physical phenomenon, the difficulty in mathematical modelling and numerical simulations, and its wide range of practical relevance. This kind of the flow is common in engineering applications, such as oil exploration, heat exchangers, cables, bridge pilings and high-rise buildings. Compared with the flow over a single bluff body, the wake interference between multiple bluff bodies gives rise to the complete change of the flow characteristics, including the vortex shedding, which leads to a very different pattern of the force acting on the cylinder. Thus, some insightful analysis into flows in such a case is highly significant for a better understanding of physics of flow as well as better applications in many engineering practices. This paper uses the lattice Boltzmann method (LBM) together with immersed boundary method (IBM) for flow past two identical circular cylinders of equal diameter in different arrangement. The aim is to shed some further lights on the force acting on cylinders, as well as the pattern of the vortex shedding.

Flow characteristics in the two-cylinder system depend on the ratio of the centre-to-centre spacing $(L)$ to the cylinder diameter $(D), L^{*}=L / D$, the alignment angle $(\alpha)$ between the line linking two cylinder centres and flow direction, as well as Reynolds number $\operatorname{Re}=u_{0} D / v$, where $u_{0}$ is the incoming free-stream velocity, and $v$ is the kinetic viscosity. Arrangements of two cylinders can be classified into three categories, including tandem $\left(\alpha=0^{\circ}\right)$, staggered $\left(0^{\circ}<\alpha<90^{\circ}\right)$ and side-by-side $\left(\alpha=90^{\circ}\right)$. Reviews of earlier work were given by Zdravkovich $(1977,1987,2003)$ and more recent ones by Sumner (2010). Broadly speaking, at sufficient small $L^{*}$, the two cylinders behave like a single bluff body, while at sufficiently large $L^{*}$, they behave like two independent bodies in the free-stream and wake interaction between two cylinders vanishes. It is within certain range of $L^{*}$, the interactions between the flows past two cylinders become highly complex. Then at a given $L^{*}$, many of these interaction features are more sensitive to $\alpha$ than to $\operatorname{Re}$ when the flow is subcritical even though it may be unstable.

For cylinders in tandem arrangement, or $\alpha=0^{\circ}$, Zdravkovich (1987) discussed some essential features for this case based on experimental evidence. These features in the wake were also observed later by many numerical simulations (Slaouti and Stansby, 1992; Mittal et al., 1997; Meneghini et al., 2001). The two cylinders can be combined as a single bluff-body when $1<L^{*}<1.2-1.8$ (Zdravkovich 1987). The free shear layer by the upstream body will 
1 pass by the downstream cylinder and the vortex shed from the two cylinders will be mainly in 2 the wake behind the downstream cylinder. The oscillation of the flow is mainly periodic with 3 period $T_{v}$. This gives the frequency $f_{v}=1 / T_{v}$, which is usually defined through the Strouhal 4 number $S t=f_{v} D / u_{0}$. Although the oscillation may have frequency components $n f_{v}$ $5 \quad(n=1,2,3 \cdots), n=1$ is usually dominant and it is usually referred as a single frequency 6 oscillation. When 1.2-1.8< $L^{*}<3.4-3.8$ (Zdravkovich, 1987), shear layer shed from the upstream body will reattach at the downstream body and a vortex street is formed only behind the downstream cylinder. The oscillation of the flow is still dominated by a single frequency. When $3<L<4$ (Zdravkovich, 2003) or $3<L \quad 5$ (Xu and Zhu, 2004), the wake pattern behind the upstream cylinder or before the downstream cylinder may intermittently change between attachment on the downstream cylinder and eddy shedding from the upstream cylinder at a higher Reynolds number, say at an order around $o\left(10^{4}\right)$, and it is referred as " bi-stable flow" (Igarashi, 1981). The oscillation of flow has two different periods and two dominant frequency components at the bi-stable regimes. This can be understood by the fact for the upstream cylinder the incoming flow is steady and uniform. The flow behind the upstream cylinder or before the downstream body is, however, oscillatory. A new kind of oscillatory flow could be generated by the second cylinder, and hence two frequency components or bi-stable flow phenomenon. When $L^{*}>3.4-3.8$ (Zdravkovich, 1987), the shed vortex will appear evidently behind each of the cylinders or co-shedding will occur. The oscillation is once again dominated by a single frequency. This could partly be due to the lock in effect (Xu and Zhu, 2004). Also at relatively lower Reynolds number, the oscillatory flow behind the upstream cylinder will decrease faster away from the cylinder because of the larger viscous effect. Thus as $L^{*}$ increases, the flow to the downstream cylinder becomes more uniform. The value of the single frequency at this range of $L^{*}$ is still different from that of an isolated cylinder. It can be expected, however, as $L^{*}$ further increases, the frequency will tend to that corresponding to a single cylinder.

For the flow past two side-by-side $\left(\alpha=90^{\circ}\right)$ cylinders, detailed discussions can be found in Bearman and Wadcock (1973), Williamson (1985), Kim and Durbin (1988), Sumner et al. (1999), Zhou et al. (2002), Kang (2003), Carini et al. (2014) and Singha et al. (2016). Sumner (2010) identified three flow regimes. When $1 \quad L \quad 1.1 \quad 1.2$, the flow behaves in a manner similar to that corresponding a single bluff-body, or "the single body case". The oscillation of 
1 the flow is dominated by a single frequency. The Strouhal number is not sensitive to $L$ 2 within this range and its value is lower than that of a single cylinder. When

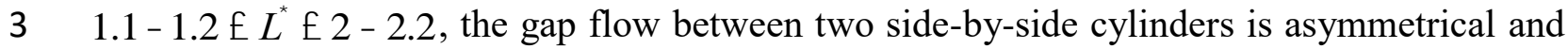
4 biased towards one cylinder. The bias may be permanent, the "deflected case" or alternate randomly, the "flip-flopping case". For deflected patterns, the developed flow is periodic and the oscillation of the flow is still dominated by a single frequency. For flip-flopping patterns, however, highly complex wake and vortex-street interaction occur. The oscillation of the flow is no longer periodic and therefore the frequency of flow oscillation is a broadband spectrum rather than discrete. When $L \quad 2$ 2.5, two parallel synchronized vortex streets are formed, which can be either symmetric about the centreline between the cylinders, the "anti-phase case", or anti-symmetric, the "in-phase case". For both of these two cases, the flow is periodic and the flow oscillation is dominated by a single frequency, whose value is close to that for an isolated cylinder.

For two staggered $\left(0^{\circ}<\alpha<90^{\circ}\right)$ cylinders, studies have been undertaken by Kiya et al. (1980), Zdravkovich (1987), Sumner et al. (2000), Jester and Kallinderis (2003), Akbari and Price (2005), Hu and Zhou (2008a, b) and Tong et al. (2015). Sumner (2010) provided a comprehensive review. The interactions involve four shear layers from the upper and lower sides of two cylinders, Karman vortex formation process as well as two Karman vortex streets. The complex nature of the interactions depends on both the spacing ratio $L^{*}$ and the alignment angle $\alpha$. It is suggested that the behaviour of the $S t$ data can be classified into three groups by spacing ratio $L^{*}$. At all $\alpha$ and small spacing with $1 \quad L \quad 1.25$ (Sumner et al., 2000), the behaviour of the flow is similar to that of a single-bluff body. At $\alpha \leq 30^{\circ}$ and $1.125 \leq L^{*} \leq 3-4$ (Sumner et al., 2000) the vortex shed by the lower cylinder is mostly suppressed, while at large spacing ratio $\left(L^{*}>3-4\right)$ (Sumner et al., 2000), in its near-wake region, an uninhibited vortex street can be formed, similar to a single, isolated circular cylinder. The oscillation at $\alpha \leq 30^{\circ}$ is dominated by a single frequency. The bi-stable case was not discussed in Sumner et al. (2000), which could be due to the fact the Reynolds number is not the same as that in Igarashi (1981). At $\alpha \geq 30^{\circ}$ and $L \quad 1.25$ (Sumner et al., 2000), the shed vortex occurs from each of the cylinders. For most but not all cases, the oscillation of the flow is dominated by two frequencies. The difference between these two frequencies becomes smaller as the distance of two cylinders becomes larger and the wake 
1 interference weakens. When $L^{*}$ further increases, the two pronounced frequency cases are 2 hardly observed and the oscillation is dominated by a single frequency. At $L$ 1.25, which covers the flip-flopping region for the side-by-side case, the oscillatory behaviour of the flow is expected to very much depend on $\alpha$. The frequency components change from some discrete values to a continuous spectrum, which is a focus of the current investigation.

The present work is based on lattice Boltzmann method (LBM). LBM is based on microscopic models and mesoscopic kinetic equations (Chen and Doolen, 1998; WolfGladrow, 2000; Aidun and Clausen, 2010). It can recover the incompressible Navier-Stokes (NS) equation based on the Chapman-Enskog expansion (Cercignani, 1988) at an error proportional to $M^{2}$, where $M=u / c_{s}$ is the Mach number, $u$ is the fluid velocity and $c_{s}$ is the sound speed (Wolf-Gladrow, 2000). Macroscopic flow properties, such as fluid velocity and pressure, can be obtained by the particle density distribution function. Because of some of its inherent advantages, LBM has been extensively and successfully used in many fluidsolid interaction problems (Li et al., 2004; Peng et al., 2006; Wu and Su, 2009; Li et al., 2016). In this work, the structure-fluid boundary is treated by the immersed boundary method (IBM). It replaces the body surface with a layer of distributed force whose value is determined by the no-slip condition. The combined IBM and LBM allow us to solve the problem in a fixed structured mesh even if the body is in motion.

We shall focus on the case with intermediate spacing of two identical cylinders. We shall undertake systematic simulations to investigate how the oscillatory behaviours of the lift force and drag change with the angle $\alpha$, as well as the flow features through vorticity contours. The Reynolds number is chosen as $R e=200$, and accordingly the spacing ratio as $L^{*}=1.5$. The work tries to show detailed frequency components or spectrum of lift and drag coefficients on both cylinders at different $\alpha$ and how the force and wake vary with $\alpha$, the change of discrete frequency components, the change from discrete frequency components to continuous spectrum. It ought to point out that the wake of a single cylinder may become noticeably three-dimensional at $\mathrm{Re}>185-200$. For the problem related two cylinders in different arrangement considered in this paper, we may refer to the work of Carmo \& Meneghini (2006). They undertook both two- and three-dimensional numerical simulations for flow past a pair of tandem cylinders at $L^{*}=1.5-8$ and $\mathrm{Re}=160-320$. It was found that when $L^{*}=1.5$ and $\operatorname{Re}<270$, values of Strouhal number from two- and three-dimensional 
1 simulations were almost same and there was no significant three-dimensional flow in the gap between the cylinders. One may then expect that the three-dimensional effect would not have major effect on the results presented in this paper for two cylinders with similar values of $L^{*}$

4 and Re.

5

6 The paper is organized as follow. In section 2 the numerical method based on LBM and IBM 7 is presented, while in section 3 validation through comparison is undertaken and results are 8 provided together with discussion and analysis. This is followed by the conclusions.

\section{Numerical method}

The flow of viscous, incompressible and Newtonian fluid is governed by the following continuity equation and Navier-Stokes (NS) equation,

$$
\nabla \cdot \vec{u}=0
$$

$$
\frac{\partial \vec{u}}{\partial t}+(\vec{u} \cdot \nabla) \vec{u}=-\frac{\nabla p}{\rho}+v \nabla^{2} \vec{u},
$$

where $\vec{u}$ is the fluid velocity, $p$ is the pressure, is the fluid density, is the kinematic viscosity. These two equations are then combined with the no-slip condition on the solid 17 surface, or

$$
\vec{u}=\vec{U}^{d}(s),
$$

where $\vec{U}^{d}$ is the velocity of the body surface.

\subsection{Lattice Boltzmann method}

22

Many numerical methods solve the macroscopic equations described in Eqs. (1) and (2) together with Eq. (3) through either discretized mesh based method or discrete point based method. LBM approaches the problem in a different manner. It is based on the microscopic model and mesoscopic kinetic equation (Succi, 2001). The method assumes that a mass distribution is function of location and velocity. The density at each location is equal to the integration of the density function with respect to the velocity. In the numerical procedure, the integration is discretized and is in the form of weighted summation. Based on this principle, the LBM governing equation can be written as (Chen and Doolen, 1998; Aidun and Clausen, 2010) 


$$
f_{i}\left(\vec{x}+\vec{e}_{i} \Delta t, t+\Delta t\right)=f_{i}(\vec{x}, t)-\frac{1}{\tau}\left(f_{i}(\vec{x}, t)-f_{i}^{e q}(\vec{x}, t)\right),
$$

2 where $f_{i}$ is the weighted density distribution function corresponding to each discretized

3 velocity $\vec{e}_{i}$, and $f_{i}^{e q}$ is the corresponding equilibrium distribution function. $\vec{x}$ in Eq. (4) is

4 the position vector in the Cartesian coordinate system $O x y$. in the equation is the 5 relaxation time, and $t$ is the time step.

6

7 For the two-dimension problem, we adopted the nine-discretized velocity, or D2Q9 model

8 (Qian et al., 1992). Corresponding to that we have $\vec{e}_{i}=c(\cos ((i-1) \pi / 2), \sin ((i-1) \pi / 2))$

$9 \quad i=1 \quad 4, \vec{e}_{i}=\sqrt{2} c(\cos ((i-5) \pi / 2+\pi / 4), \sin ((i-5) \pi / 2+\pi / 4)) \quad i=5 \quad 8$, and $\vec{e}_{0}=(0,0)$.

10 The equilibrium distribution function is of the form

$$
f_{i}^{e q}(\vec{x}, t)=\rho \omega_{i}\left[1+\frac{\vec{e}_{i} \cdot \vec{u}}{c_{s}^{2}}+\frac{\left(\vec{e}_{i} \cdot \vec{u}\right)^{2}}{2 c_{s}^{4}}-\frac{u^{2}}{2 c_{s}^{2}}\right],
$$

where weighting coefficient ${ }_{i}$ are given as ${ }_{0}=4 / 9,{ }_{i}=1 / 9$ for $i=14$, and

$$
{ }_{i}=1 / 36 \text { for } i=58 \text {. }
$$

15 The fluid domain is then discretized by the structured mesh with $x=y=c t=h$, where

$16 c=\sqrt{3} c_{s}$ is the lattice speed. The solution of Eq. (4) is then obtained through the streaming and collision process (Wolf-Gladrow, 2000). From the density distribution function, the fluid density and the fluid velocity at each point can be respectively calculated as follow

$$
\begin{gathered}
\rho=\sum_{i=0}^{8} f_{i}, \\
\rho \vec{u}=\sum_{i=0}^{8} f_{i} \vec{e}_{i} .
\end{gathered}
$$

According to the Chapman-Enskog expansion (Cercignani, 1988) the above LBM equation can recover the NS equations with the kinematic viscosity

$$
=(0.5) c_{s}^{2} t
$$


1 and the order of accuracy is proportional to $\left(u / c_{s}\right)^{2}$ (Wolf-Gladrow, 2000).

2

3

4

5

6

\subsection{Immersed boundary method}

Eq. (4) may be equivalent to Eqs. (1) and (2). However, we still need to impose the no-slip condition on the body surface in Eq. (3). Here we adopt the immersed boundary method (Peskin, 1977). The method has been used extensively and refined continuously in more recent years (Lai \& Peskin, 2000; Lima et al., 2003; Wu \& Shu, 2009). The principle of the method is to add a distribution of force $\vec{g}$ into Eq. (2) or

$$
\frac{\partial \vec{u}}{\partial t}+(\vec{u} \cdot \nabla) \vec{u}=-\frac{\nabla p}{\rho}+v \nabla^{2} \vec{u}+\vec{g} .
$$

From Eq. (2), the updated velocity $\vec{u}^{*}$ without the forcing term can be obtained from

$$
\frac{\partial \vec{u}}{\partial t}=\frac{\vec{u}^{*}(\vec{x}, t+\Delta t)-\vec{u}(x, t)}{\Delta t}=-(\vec{u} \cdot \nabla) \vec{u}-\frac{\nabla p}{\rho}+v \nabla^{2} \vec{u}=R S .
$$

From Eq. (9), we have

$$
\frac{\vec{u}^{d}(\vec{x}, t+\Delta t)-\vec{u}(\vec{x}, t)}{\Delta t}=R S+\vec{g}(\vec{x}, t)
$$

where $\vec{u}^{d}$ is the desired fluid velocity. From Eqs. (10) and (11), the external force should be obtained from

$$
\vec{g}(\vec{x}, t)=\frac{\vec{u}^{d}(\vec{x}, t+\Delta t)-\vec{u}^{*}(\vec{x}, t+\Delta t)}{\Delta t} .
$$

Obviously, $\vec{g}$ cannot be applied in Eq. (9) everywhere as the equation has to be equivalent to Eq. (2) in the fluid domain. The forcing term should be applied only to a strip along the body surface $\vec{X}(s, t)$, including the pressure and shear force. As velocity is given on each lattice node, the velocity $\vec{U}(s, t)$ on the boundary without the forcing term can be obtained by the delta function ${ }_{h}$

$$
\vec{U}(s, t)=\sum_{\vec{x}} \vec{u}^{*}(\vec{X}, t) \delta_{h}(\vec{X}-\vec{X}(s, t)),
$$

which is the discretized form of the integration involving Dirac delta function. Based on Peskin (2002), the delta function $\delta_{h}(\vec{x})$ can be written as follow 


$$
\delta_{h}(\vec{x})=\delta_{h}(x) \delta_{h}(y)
$$

2 and

$$
{ }_{h}(r)=\left\{\begin{array}{ll}
\frac{1}{4 h}\left(1+\cos \left(\frac{|r|}{2 h}\right)\right) & |r| 2 h \\
0 & \text { otherwise }
\end{array} .\right.
$$

5 The desired velocity $\vec{u}^{d}$ on the body surface should be body surface velocity $\vec{U}^{d}$. Thus, the required body force on the solid boundary should be

8 Corresponding to that the external force in the strip next to the body surface should be 9 obtained from

$$
\vec{g}(\vec{X}, t)=\sum_{s} \vec{G}(s, t) \delta_{h}(\vec{X}-\vec{X}(s, t)) .
$$

To combine this IBM with the present LBM method, Eq. (4) can be modified as

$$
f_{i}\left(\vec{x}+\vec{e}_{i} \Delta t, t+\Delta t\right)=f_{i}(\vec{x}, t)-\frac{1}{\tau}\left(f_{i}(\vec{x}, t)-f_{i}^{e q}(\vec{x}, t)\right)+\Delta t \frac{w_{i} \rho}{c_{s}^{2}} \vec{e}_{i} \cdot \vec{g},
$$

which can be found to be equivalent to Eq. (9) in the manner that Eq. (4) is equivalent to Eq. (2).

\section{Numerical results and discussions}

\subsection{Convergence and comparison}

The flow past a fixed cylinder at Reynolds number $\mathrm{Re}=200$ has been carried out in order to verify the numerical method and the gird convergence.The incoming flow is from the left hand side of the body. The cylinder is located in the flow field. The computational domain is taken as $L e=10 D, L s=10 D$ and $L r=20 D$, as shown in Fig.1(a). A Dirichlet boundary condition $\left(\vec{u}=\left(u_{0}, 0\right)\right)$ is adopted at the inflow and far-field boundaries. The uniform steady flow is used in the initial condition. In the present LBM, Mach number is $M=0.1$. A grid paramenter is defined by $s=D / h$ for simplicity. The nondimensional time $T=u_{0} t / D$ is 
1 used in the rest of the paper. For analyses, the fluctuating force history is collected for a

2 sufficiently long period of time with $\mathrm{T}>1200$.

3

4 The $F_{D}$ and $F_{L}$ are drag and lift forces on the body, respectively. The corresponding 5 coefficients are defined by $C_{D}=F_{D} / 0.5 \rho u_{0}^{2} D$ and $C_{L}=F_{L} / 0.5 \rho u_{0}^{2} D$. Their results with 6 Strouhal number are shown in Table 1, which indicates that the grid parameter $s=70$ is 7 sufficient to obtain convergent solution. The table also provides the comparison between the 8 present results and those obtained by experiment and other numerical simulations. A good 9 agreement can be found, suggesting that the present numerical method is accurate and resolution is adequate. Vortex contour of this case are given in Fig. 2, which shows the oscillatory flow of the wake. The temporal variations of the drag and lift coefficients are shown in Fig. 3. Fourier analysis of the periodic drag and lift coefficients is shown in Fig. 4. We may observe that the periodic oscillation gives virtually a single frequency. The Strouhal number corresponding to the lift coefficient is 0.207 , and that of drag coefficient is 0.414 . The latter is the double of the former. In fact when the body is symmetric and the flow pattern shown in Fig. 2 is periodic in time, the flow at $y>0$ and $T+T_{v} / 2$ should be the mirror image of the flow at $y<0$ and $T$. Using this, it can be shown (Wu, 2000) that the lift coefficient $C_{L}$ has frequency components of $(2 n+1) S t$ only while the drag coefficient $C_{D}$ has $2 n S t(n=0,1 \cdots)$.

(a)

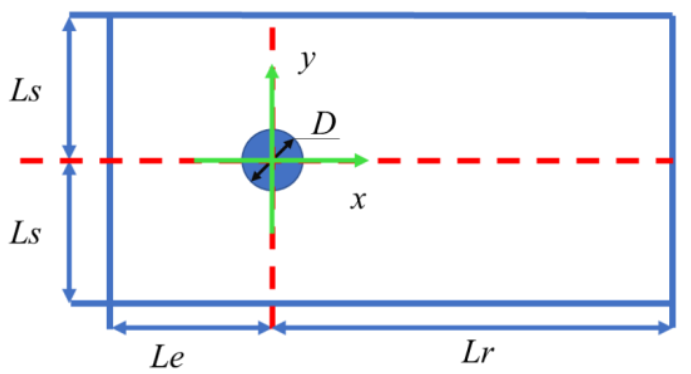

(b)

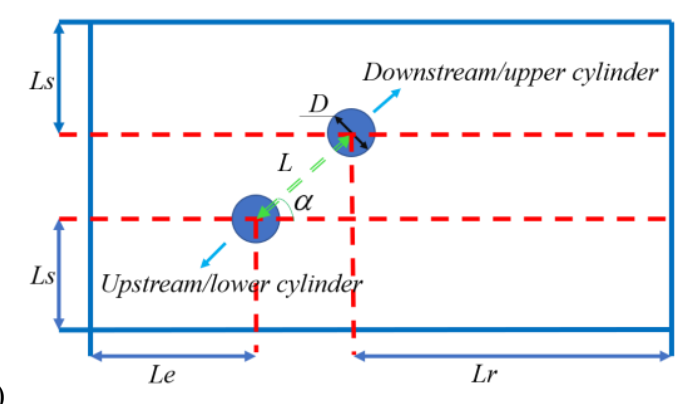

Figure 1. Computational configurations for (a) a single cylinder and (b) two cylinders. 
1 Table1.

2 Drag and lift coefficients and Strouhal number on a single cylinder at $\mathrm{Re}=200$

\begin{tabular}{|c|c|c|c|c|c|}
\hline & \multicolumn{2}{|l|}{$C_{D}$} & \multicolumn{2}{|l|}{$C_{L}$} & \multirow[t]{2}{*}{ St } \\
\hline & Max. & Min. & Max. & Min. & \\
\hline \multicolumn{6}{|l|}{ Present result: } \\
\hline $\mathrm{s}=50$ & 1.338 & 1.252 & 0.569 & -0.569 & 0.206 \\
\hline $\mathrm{s}=70$ & 1.333 & 1.250 & 0.569 & -0.569 & 0.207 \\
\hline $\mathrm{s}=98$ & 1.334 & 1.251 & 0.569 & -0.569 & 0.207 \\
\hline Wu \& Hu (2006) & 1.385 & 1.324 & 0.563 & -0.564 & 0.190 \\
\hline Chan \& Anastasiou (1999) & 1.50 & 1.42 & 0.70 & -0.70 & 0.23 \\
\hline Chen et al. (1999) & 1.37 & 1.29 & 0.72 & -0.72 & 0.20 \\
\hline Braza et al. (1986) & \multicolumn{4}{|c|}{1.35 (mean) } & 0.20 \\
\hline Williamson (1996) & & & & & 0.198 \\
\hline
\end{tabular}

3

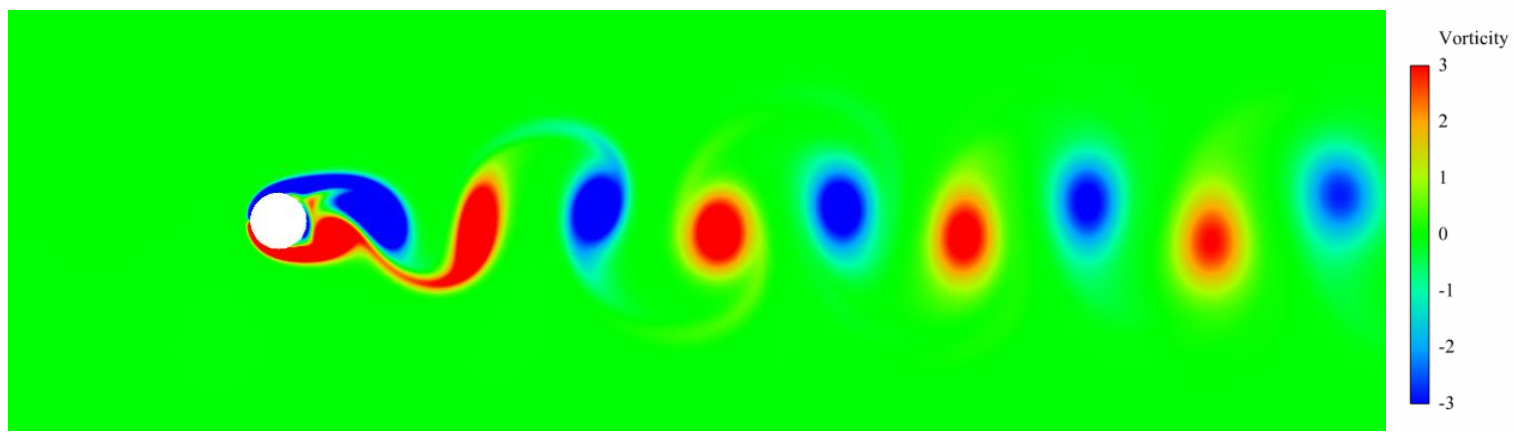

5

Figure 2. Vortex contour for a single cylinder at $\mathrm{T}=185.7$ with $\mathrm{Re}=200$

6

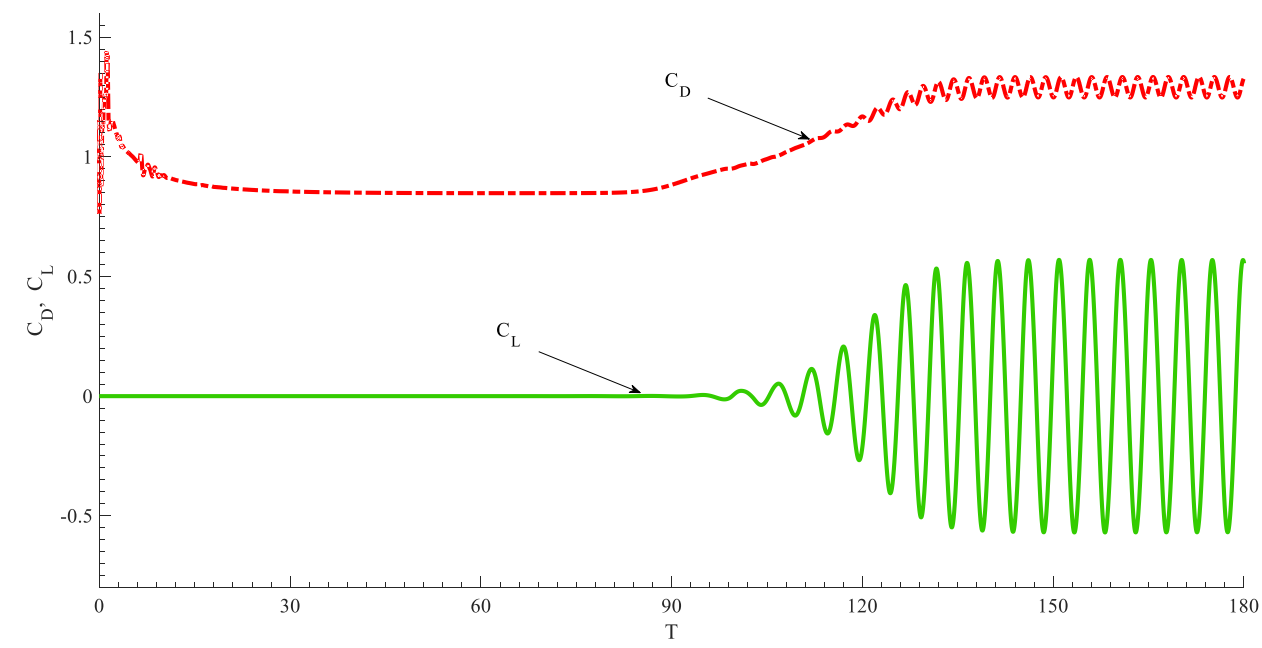

7

Figure 3. Drag and lift coefficients on a single cylinder at $\mathrm{Re}=200$ 


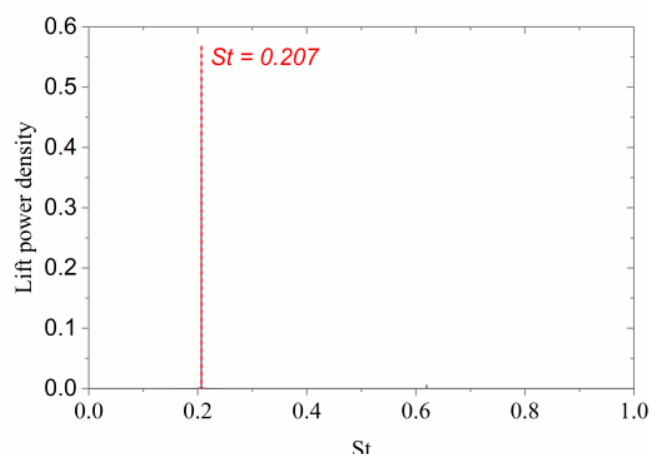

(a)

Figure 4. Power spectra of (a) the lift and (b) drag coefficients on a single cylinder with $\mathrm{Re}=200$

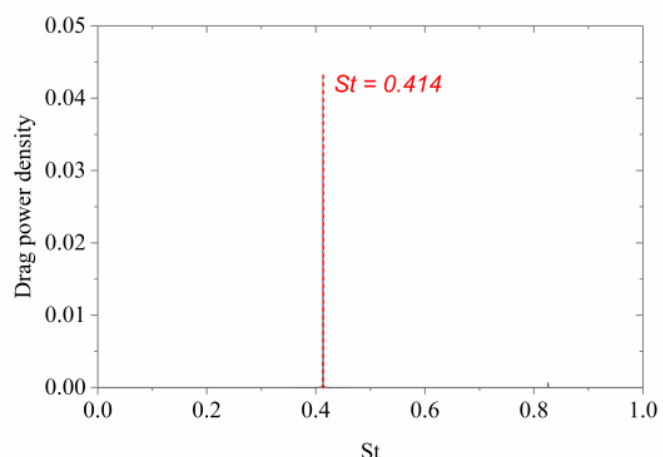

(b)

(a) 3

\subsection{The flow past two stationary cylinders in various arrangements}

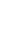

In this section, we will analyse the fluctuating force acting on individual cylinders at the fixed centre-to-centre spacing $L^{*}=L / D=1.5$ and Reynolds number $\operatorname{Re}=200$. The arrangement of the cylinders will vary with the alignment angle $\alpha$. The computational configuration for two cylinders is shown in Fig. 1(b).

The effect of domain size is first investigated. Two domains with (1) $L e=10 D, L s=10 D$ and $L r=20 D$; (2) $L e=14 D, L s=12 D$ and $L r=28 D$, respectively, are used to solve the flow past pair cylinders in tandem. Lift and drag coefficients together with Stouhal number obtained with grid parameter $s=70$ and Mach number $M=0.1$ are given in Table 2. Results from two domain sizes are virtually identical, the largest relative error is about $0.47 \%$. This establishes the adequacy of domain size (1) in computing the flow past two stationary cylinders in various arrangements. Moreover, computations are carried out for two different values of grid parameter s whose alternation changes both grid size and the time, to primarily confirm the mesh convergence for the case of two cylinders. The relative difference in mean $\mathrm{Cd}$, maximum $\mathrm{Cl}$ and $\mathrm{St}$ of both tandem cylinders obtained with $\mathrm{s}=70$ and 98 shown in Table 2 is about $0.1 \%$. Convergence with time step through Mach number are also conducted with $\mathrm{Ma}=0.1$ and 0.05 in Table 2 and excellent agreement between the two sets of results has been found. In the subsequent calculations, $\mathrm{s}=70$ and $\mathrm{M}=0.1$ are used.

Fig. 5 displays the mean drag and lift coefficients on two cylinders with the alignment angle $\alpha$. Note that all the length of the time over which the mean is obtained is the same as that used to get corresponding spectra of forces. It can be seen that with the increase of $\alpha$, mean drag coefficients on both cylinders also increase. Compared with downstream/upper cylinder, 
1 the increase of mean drag coefficient on the upstream/lower cylinder is quicker, especially at

2 smaller alignment angle $\alpha \leq 30^{\circ}$. Furthermore, with the increase of $\alpha$, the mean lift

3 coefficient on the downstream/upper cylinder increases first and then decreases rapidly, while

4 the upstream/lower cylinder has the opposite trend.

5

6 Table2.

7 Drag and lift coefficients, and Strouhal number for two tandem cylinders (Indexes 1 and 2 represent downstream 8 and upstream cylinder, respectively.)

\begin{tabular}{ccccccccc}
\hline Domain & $\mathrm{s}$ & $\mathrm{M}$ & $\overline{\mathrm{C}}_{\mathrm{D} 1}$ & $\overline{\mathrm{C}}_{\mathrm{D} 2}$ & $\begin{array}{c}\text { Max. } \\
\mathrm{C}_{\mathrm{L} 1}\end{array}$ & $\begin{array}{c}\text { Max. } \\
\mathrm{C}_{\mathrm{L} 2}\end{array}$ & $\mathrm{St}_{1}$ & $\mathrm{St}_{2}$ \\
& & & & & & \\
\hline$(1)$ & 70 & 0.1 & -0.1577 & 1.0209 & 0.0753 & 0.0213 & 0.168 & 0.168 \\
$(2)$ & 70 & 0.1 & -0.1579 & 1.0206 & 0.0753 & 0.0212 & 0.168 & 0.168 \\
$(1)$ & 98 & 0.1 & -0.1578 & 1.0209 & 0.0753 & 0.0213 & 0.168 & 0.168 \\
$(1)$ & 70 & 0.05 & -0.1577 & 1.0208 & 0.0753 & 0.0213 & 0.168 & 0.168 \\
\hline
\end{tabular}

9

10

12

13

14

15 (a)

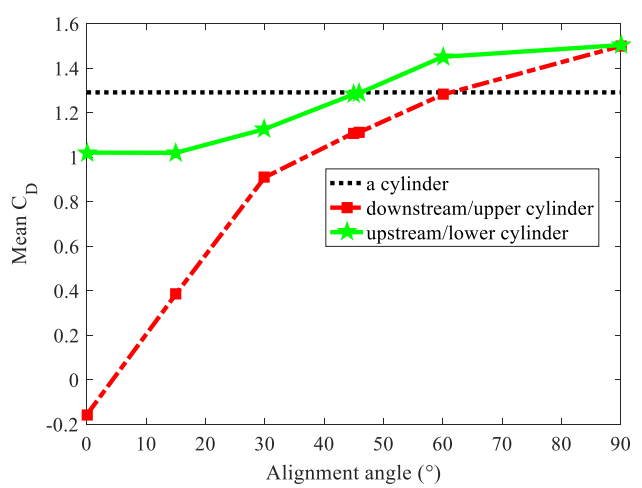

Fig.5 Mean drag and lift coefficients as functions of $\alpha$ (b)

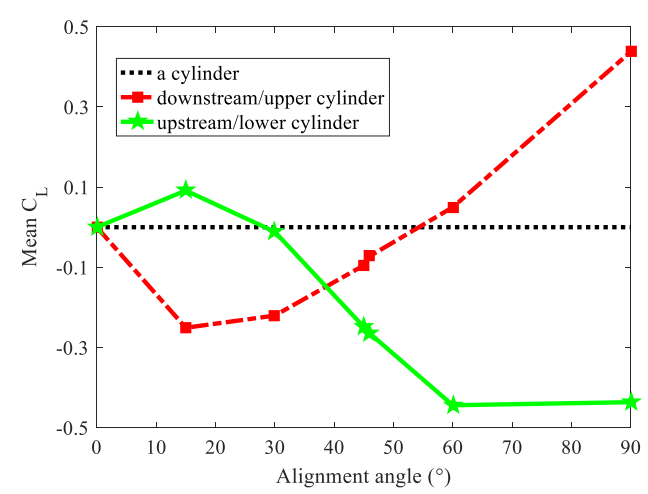

\subsubsection{The flow past two tandem cylinders}

Force coefficient time histories in Fig. 6. The amplitude of the lift coefficient, and the mean and amplitude of drag coefficient for both cylinders are much smaller than that for an isolated cylinder, as a result of the vortex shedding suppression from the downstream cylinder. The drag coefficient is positive for the upstream cylinder and negative for the downstream cylinder, and thus there is an attractive force between two cylinders. These phenomena are consistent with that observed in the experiment by Zdravkovich (1987). The lift and drag coefficients on both cylinders here are almost in phase. The lift coefficients as well as drag coefficients on both cylinders here are almost in phase. The main reason may be that the 
1 vortex shed from the upstream cylinder is suppressed by the downstream cylinder and there is only one main vortex street behind two cylinders, shown in Fig. 8. Moreover, the configuration in tandem is symmetric about $\mathrm{x}$ axis and two shear layers shed from the upstream cylinder reattach at the downstream cylinder, shown in Fig. 8.

6 Spectrum analysis of the force coefficients is undertaken and the results are given in the 7 Fig.7. The lowest frequency can be defined as $f_{v 0}=1 / T_{v}$, which is the dominant frequency 8 for the lift forces on both cylinders at the corresponding Strouhal number $S t_{0}=0.168$, as can 9 be seen in Figs. 7(a) and 7(c). There is also a minor component at $3 S t_{0}=0.504$ and beyond that there is nothing further visible. The drag coefficients for both cylinders have a dominant component at $2 S t_{0}=0.336$ together with a much smaller component at $4 S t_{0}=0.672$. This follows the pattern that the $C_{L}$ on both cylinders has components of $(2 n+1) S t(n=0,1)$, while the $C_{D}$ of both cylinders has $2 n S t(n=1,2)$, in which the steady component of $n=0$ has been taken out. The force coefficient amplitude in the two tandem cylinder case is smaller than that on an isolated cylinder. This can be partly explained by vorticity contour in Fig. 8 . The oscillatory behaviour of the flow behind the upstream cylinder is much weaker than that in Fig.2 for the single cylinder. The Karman vortex street is not fully developed behind the upstream cylinder and in front of the downstream cylinder. This leads to a smaller oscillatory force on the upstream cylinder. For the downstream cylinder, there are oscillatory flows on both sides, although it is stronger behind the cylinder and weaker before the cylinder. Also, the incoming velocity to the downstream cylinder is lower than the free stream due to the "blockage" effect of the upstream cylinder. This leads to a smaller oscillatory force.

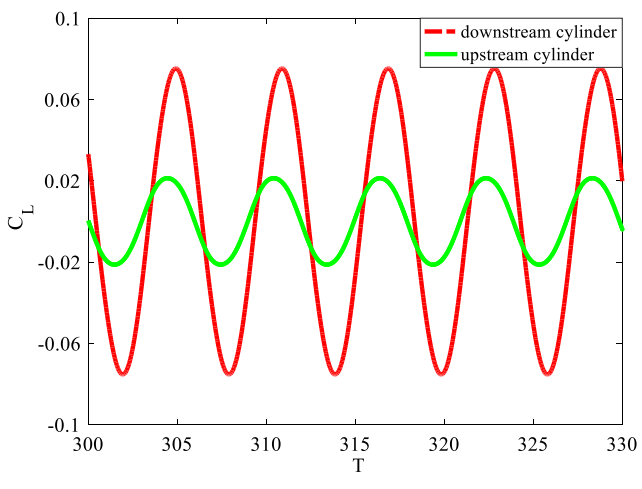

(b)

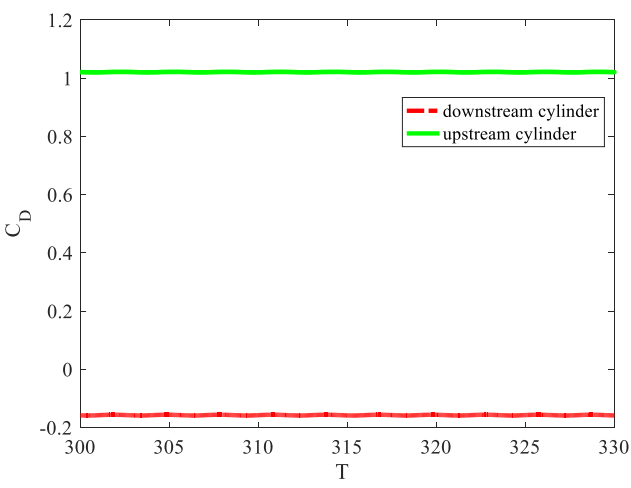

Figure 6. Force coefficient time histories on two cylinders with $\alpha=0^{\circ}$ 


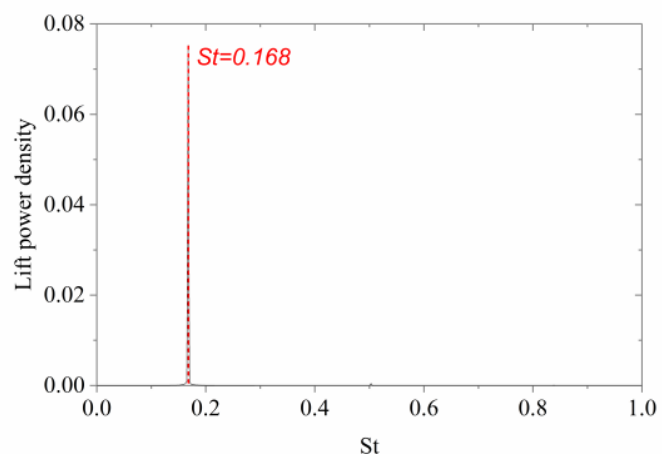

(a)

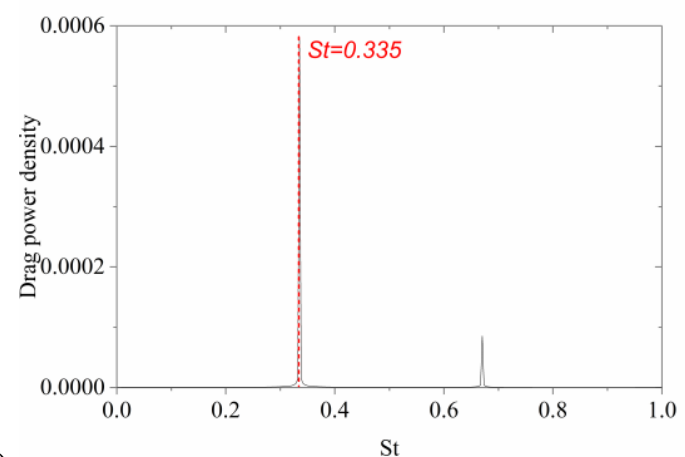

(b)

3

4

5

6

7

8

$$
9
$$

10

11

12

13

14 both lift and drag will have components $n S t_{15}(n=0,1,2 \cdots)$. The mean and maximum value

15 of the lift coefficient for the upper cylinder are negative while that of the lower cylinder is

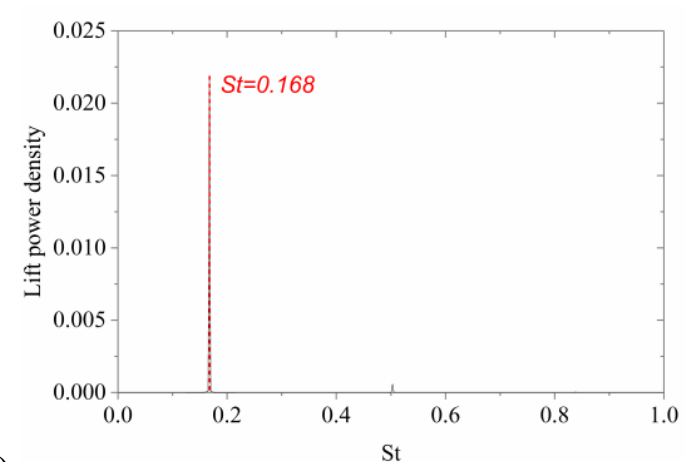

(c)

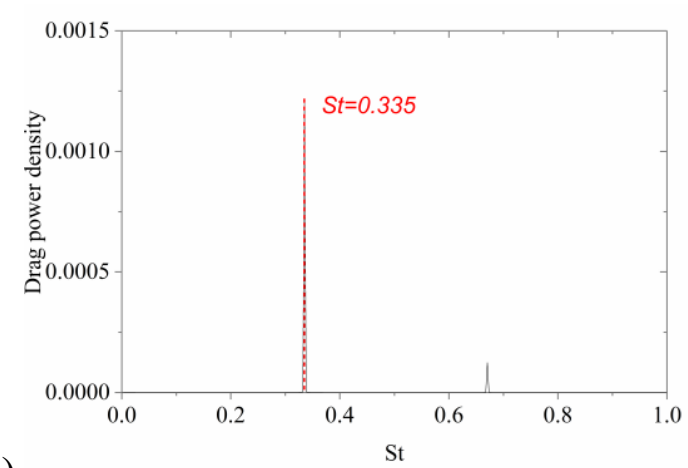

(d)

Figure 7. Spectra of force coefficients for (a)-(b) downstream cylinder; (c)-(d) upstream cylinder with $\alpha=0^{\circ}$

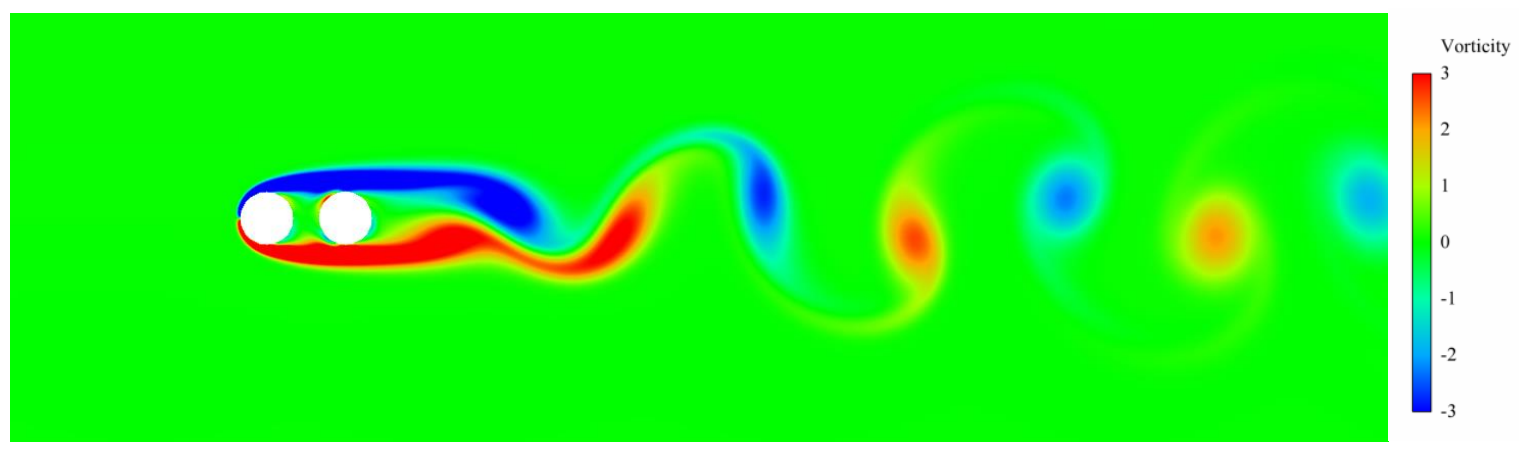

Figure 8. Vortex contour at $\mathrm{T}=273$ with $\alpha=0^{\circ}$

3.2.2 The flow past two staggered cylinders with $\alpha=15^{\circ}$

The lift and drag coefficients at $\alpha=15^{\circ}$ are shown in Fig. 9. The oscillation of the flow here is still periodic as that in the case of $\alpha=0^{\circ}$, but the detailed flow characteristics are different between these two cases. As the configuration no longer has a symmetry line as in the tandem case, around which the flow oscillates, the mean value of the lift coefficient is non-zero and positive. Even though the drag coefficient for the lower cylinder is still much higher than that 
1 for the upper cylinder, $C_{D}$ on both cylinders is positive. The lift coefficients on both cylinders 2 here are nearly in phase, which is similar to that in tandem. The main reason may be that 3 there is also only one main vortex street behind two cylinders, shown in Fig.11. However, the 4 drag coefficients at $\alpha=15^{\circ}$ are almost anti-phase. Part of the reason may be due to that 5 compared with cylinders in tandem in Fig.8, the configuration at $\alpha=15^{\circ}$ is no longer 6 symmetric about $\mathrm{x}$ axis. The downstream cylinder is higher than that of the upstream 7 cylinder, and thus only the inner shear layer from the lower cylinder is deflected into the gap 8 between the two cylinders and attached at the upper cylinder, shown in Fig.11.

Frequency spectra of lift and drag coefficients for both cylinders at $\alpha=15^{\circ}$ are displayed in

Fig. 10. The lowest frequency is $S t_{15}=0.16$, which is also the dominant frequency component. It is interesting to see that this dominant frequency is the same for both lift and drag, while in the $\alpha=0^{\circ}$ case, the component of the drag coefficient is at double lowest frequency. There are also two visible components at $2 S t_{15}=0.32$ and $3 S t_{15}=0.48$, respectively, but their amplitudes are much smaller. The lowest frequency $S t_{15}$ here is close to that for $\alpha=0^{\circ}$. This can be partly explained by vortex contour in Fig. 11. Even though the inner shear layer from the lower cylinder is deflected into the gap between two cylinders, it is constrained by the upper cylinder and thus there is only main Karman vortex street behind two cylinders. This is similar to that in the tandem case. On the other hand, compared with the vortex contour for the tandem case, the mean position of each oscillatory streamline here is moved upwards with the upper cylinder.

(a)

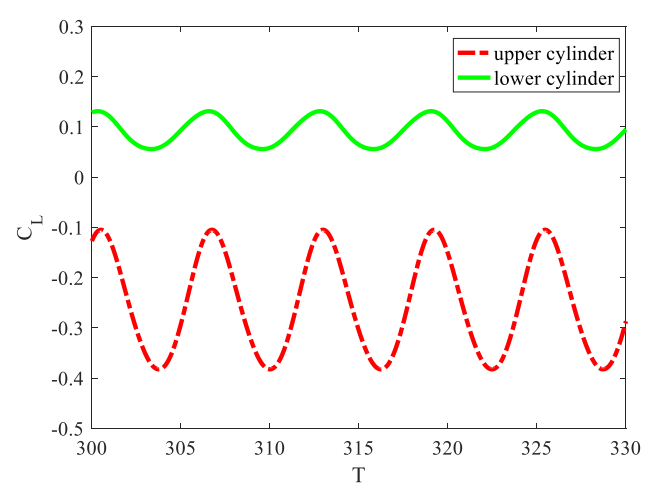

(b)

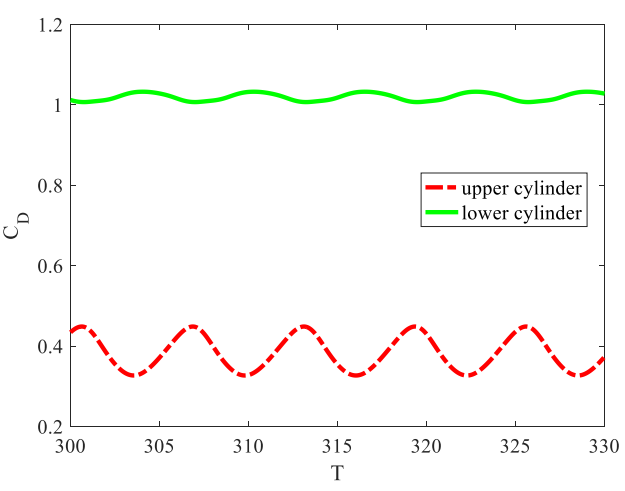

Figure 9. Force coefficient time histories on two cylinders with $\alpha=15^{\circ}$ 


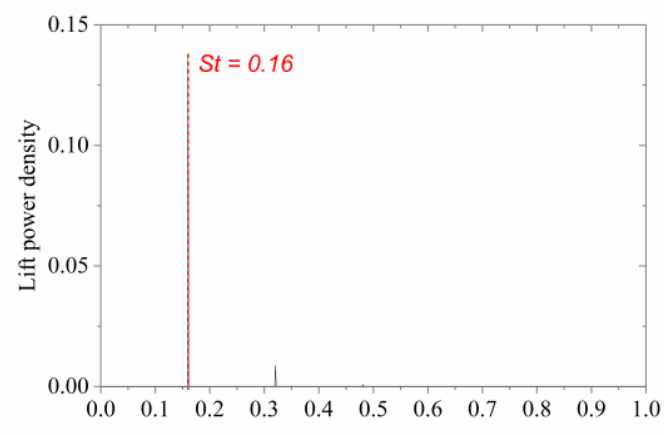

1

(a)

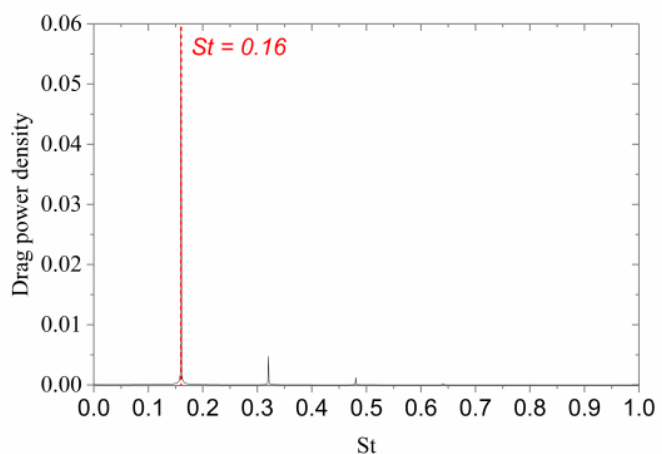

(b)

Figure 10. Spectra of force coefficient for (a)-(b) upper cylinder and (c)-(d) lower cylinder with $\alpha=15^{\circ}$

4

5

6

7

8

9

10

11

12

13

14

15 16 forces on the two cylinders here becomes smaller than that at $\alpha=15^{\circ}$. The oscillation of lift

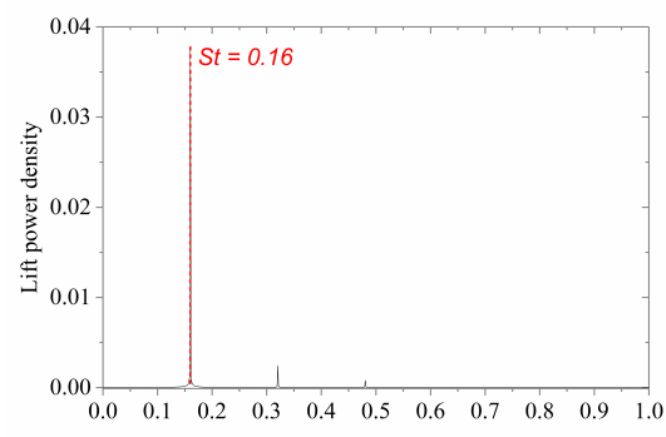

(c)

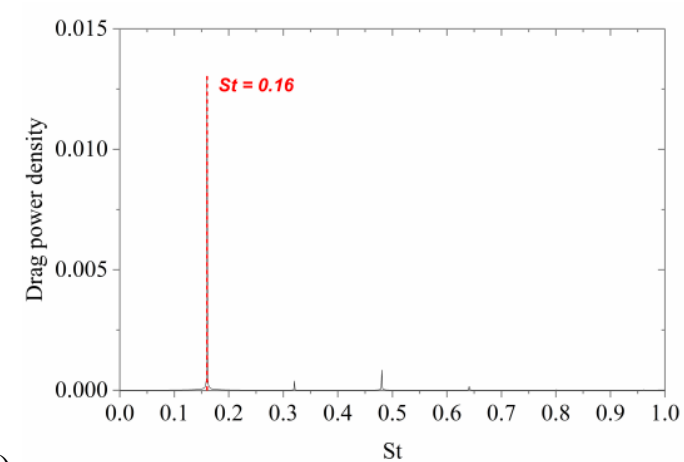

(d)

\section{3} .

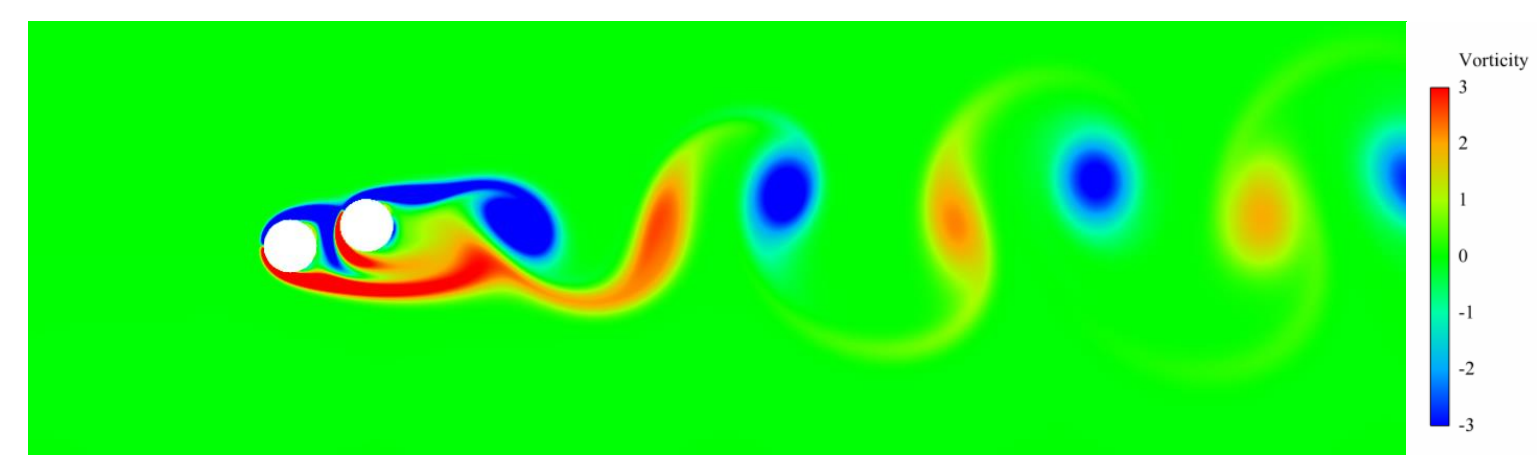

Figure 11. Vortex contour at $\mathrm{T}=273$ with $\alpha=15^{\circ}$

\subsubsection{The flow past two staggered cylinders with $\alpha=30^{\circ}$}

Lift and drag coefficient time histories on both cylinders at $\alpha=30^{\circ}$ are shown in Fig. 12 .

1 Compared with that at $\alpha=15^{\circ}$, the oscillation of the lift and drag coefficients on two 12 cylinders is less sinusoidal. The mean value for the upper cylinder is negative, same as that in 3 the $\alpha=15^{\circ}$ case and the mean result for the lower cylinder is close to zero, which is the 4 result when the configuration is symmetry, including the single cylinder and two cylinders in 5 tandem arrangements. At the same time, the difference between the mean values of drag 
1 coefficients of the two cylinders are in phase and those of the drag coefficients are anti-phase,

2 which is similar to that at $\alpha=15^{\circ}$.

3

4 Spectra of the force coefficients on each individual cylinder are given in Fig. 13. The force 5 history is periodic with respect to time, which is the same as that in the previous two cases.

6 The lowest frequency $S t_{30}=0.067$ becomes much smaller and is less than half of $7 S t_{0}=0.168$ at $\alpha=0^{\circ}$ and $S t_{15}=0.16$ at $\alpha=15^{\circ}$. However, the amplitude at the lowest 8 frequency is no longer dominant. The largest peak here is at $2 S t_{30}=0.133$, which is in fact 9 much closer to $S t_{15}=0.16$ at the $\alpha=15^{\circ}$ case. More interestingly, at $\alpha=30^{\circ}$ here, more 10 frequency components $(n=1,2,3 \cdots)$ become visible and significant, especially for the drag 11 coefficient on the upper cylinder. Fig. 14 shows vortex contour. Compared with those at $\alpha=0^{\circ}$ and $\alpha=15^{\circ}$, the position of the bottom side of the upper cylinder is higher than that of the lower cylinder axis.

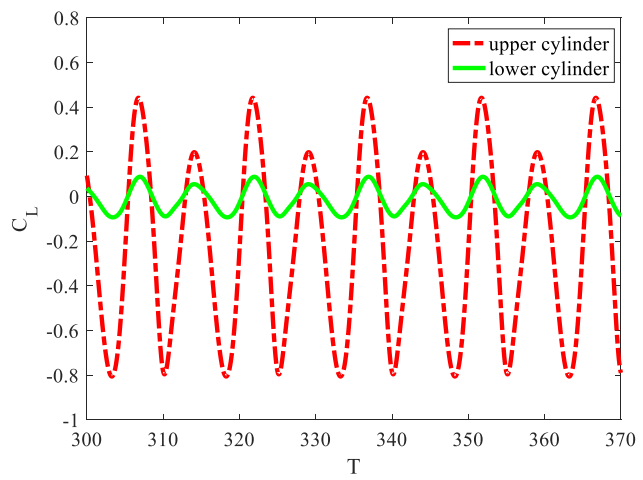

Figure 12. Force coefficient time histories on two cylinders with $\alpha=30^{\circ}$

(b)

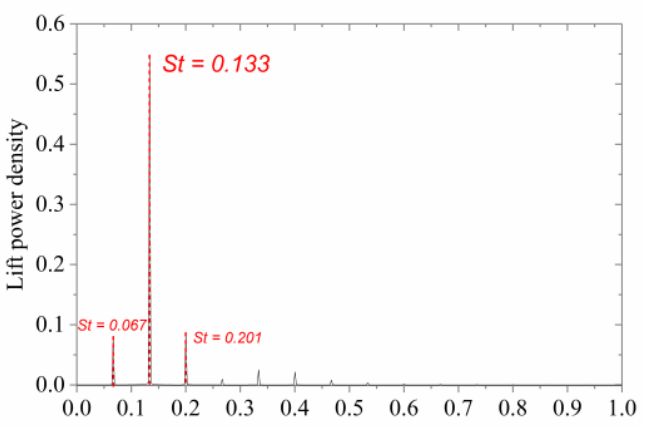

(a)
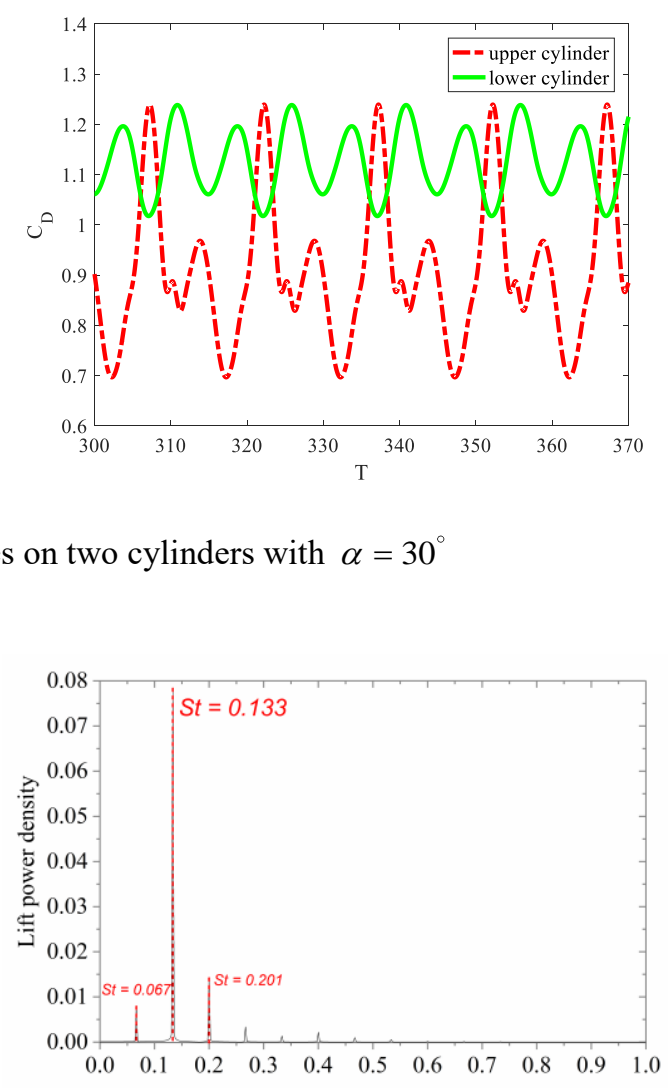

(c) 


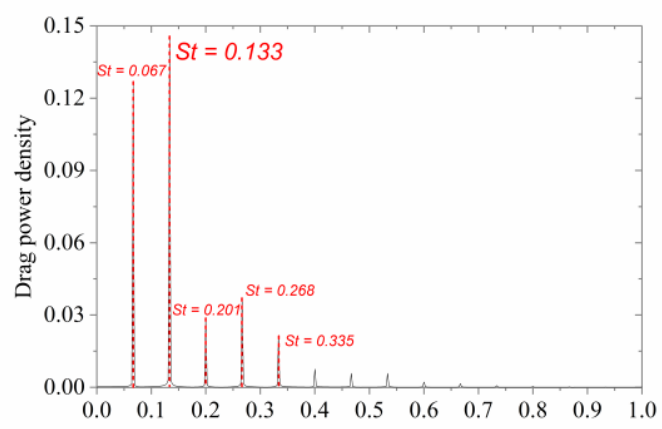

(b)

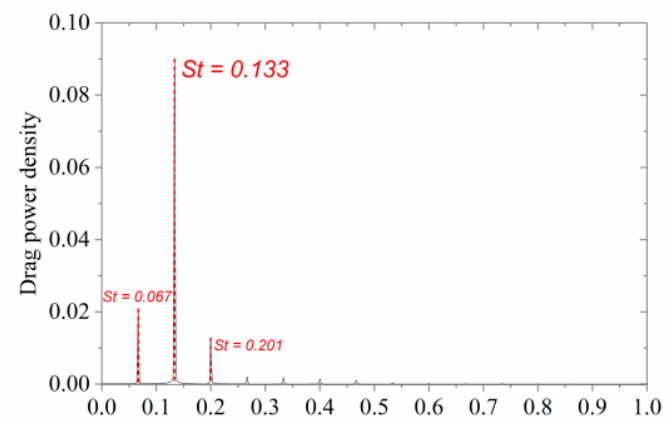

(d)

Figure 13. Spectra of force coefficients for (a)-(b) upper cylinder and (c)-(d) lower cylinder with $\alpha=30^{\circ}$

4

6

7

8

9

10

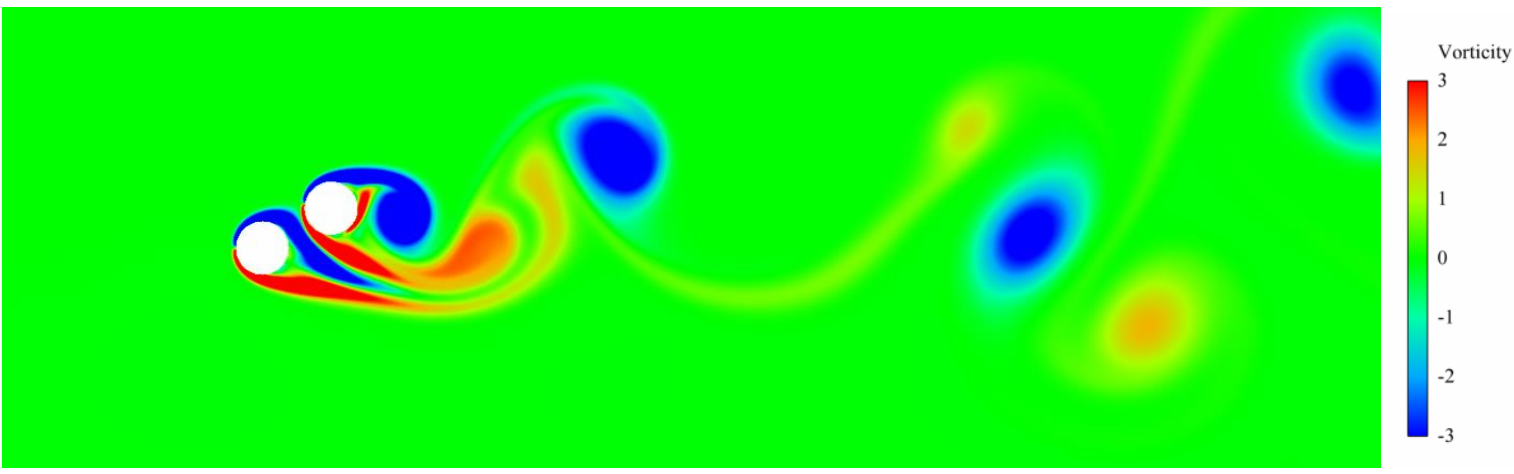

Figure 14. Vortex contour at $\mathrm{T}=273$ with $\alpha=30^{\circ}$

\subsubsection{The flow past two stationary staggered cylinders with $\alpha=45^{\circ}$}

Lift and drag coefficients on both cylinders at $\alpha=45^{\circ}$ are shown in Fig. 15. The oscillation of the lift and drag coefficients on two cylinders here is far less sinusoidal than that in the $\alpha=30^{\circ}$ case. The mean lift coefficient for the lower cylinder becomes once again non-zero, which is different from the case of $\alpha=30^{\circ}$. The mean values of both lift coefficients are found to be negative. The oscillations of two lift coefficients are nearly in phase, the same as what has been observed in the previous three cases. However, the drag coefficients are no longer synchronized and they are neither in-phase nor anti-phase. In addition, together with Figs. 6, 9 and 12, it can be found that with the increasing $\alpha$, the mean drag of the upper cylinder (the downstream cylinder) changes from the negative value to the positive and increases rapidly, while that of the lower cylinder (the upstream cylinder) increases more slowly. In other words, the alignment angle $\alpha$ has much stronger influence on the mean drag force of the upper cylinder than that of the lower cylinder. 
1 Spectra of force coefficients on each individual cylinder are given in Fig. 16. At $\alpha=45^{\circ}$,

2 frequency components are much more widely spread and a large number of components

3 become important at frequency spectra. This shows that the force coefficients become far less

4 sinusoidal in time. Furthermore, the lowest frequency $S t_{45}=0.017$ becomes much lower and

5 is less than a quarter of the lowest frequency $S t_{30}=0.067$ at $\alpha=30^{\circ}$. The lowest frequency is

6 no longer the dominant component, which is similar to that in the $\alpha=30^{\circ}$ case. The

7 amplitude peak of both drag coefficients is at $4 S t_{45}=0.068$. Different from two previous

8 staggered cases, the dominant frequencies of the lift coefficients of two cylinders are no

9 longer the same. It is at $8 S t_{45}=0.136$ for the lift coefficient on the upper cylinder, which is

10 close to $2 S t_{30}=0.133$ at $\alpha=30^{\circ}$, while it is at $4 S t_{45}=0.068$ for the lower cylinder, which is

11 the same as that of the drag coefficients of the two cylinders. To have some insights into the

12 reason for different dominant frequencies of the two lift forces, the velocity component in the

$13 y$-direction at four points $(7.5 \mathrm{D}, 12 \mathrm{D}),(7 \mathrm{D}, 10.2 \mathrm{D}),(6.2 \mathrm{D}, 9.8 \mathrm{D})$ and $(6.2 \mathrm{D}, 9.3 \mathrm{D})$ are

14 investigated. Fig. 17 gives their time histories and the corresponding frequency spectra. For

15 two points at lower and upper shear layers of the lower cylinder, the dominant frequency is

16 the same and at $4 S t_{45}=0.068$. For the upper cylinder, the dominant frequency of the velocity

17 at the upper free shear layer is at $8 S t_{45}=0.136$, while the dominant frequency at the lower

18 free shear layer is at $4 S t_{45}=0.068$, which is the same as that of two free shear layers from the

19 lower cylinder. Thus, the dominant frequency of the lift coefficient of the upper cylinder is related to that of its upper shear layer, while the dominant frequency of all other force coefficients is related to that of the other three shear layers. This is in fact consistent with what was observed in the experiment by Sumner et al. (2000). Fig. 18 shows vortex contour.
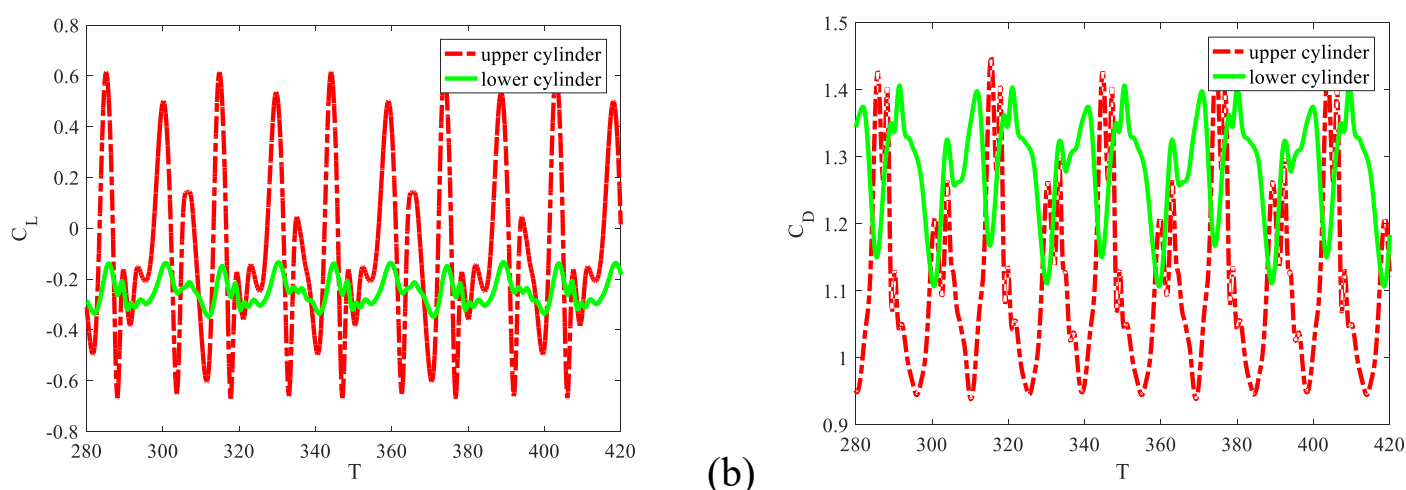

(b) 
2
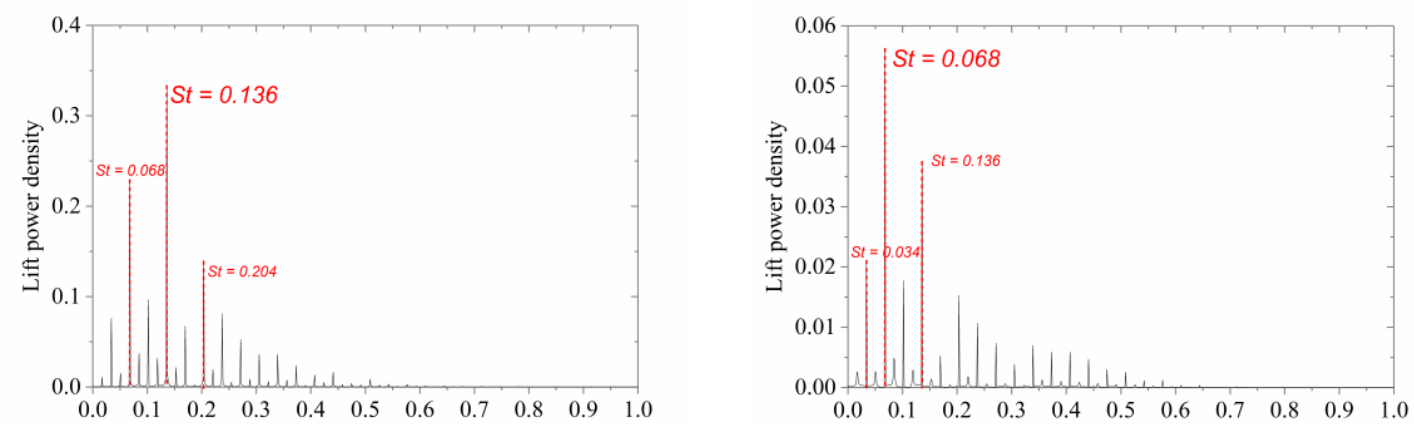

3

(a)

St

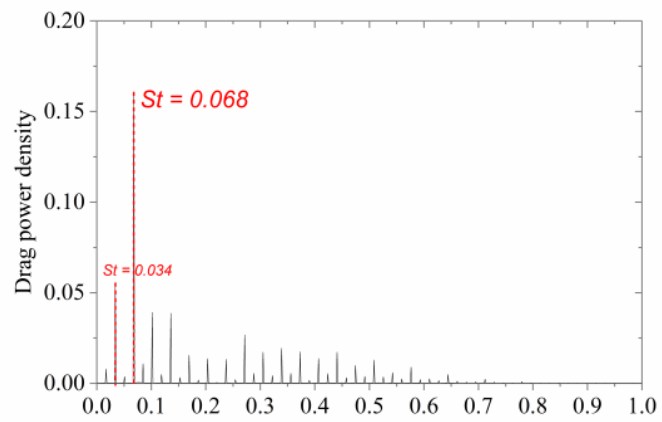

(b)

St

Figure 16. Spectra of force coefficients for (a)-(b) upper cylinder and (c)-(d) lower cylinder with $\alpha=45^{\circ}$ 6

7

(a)
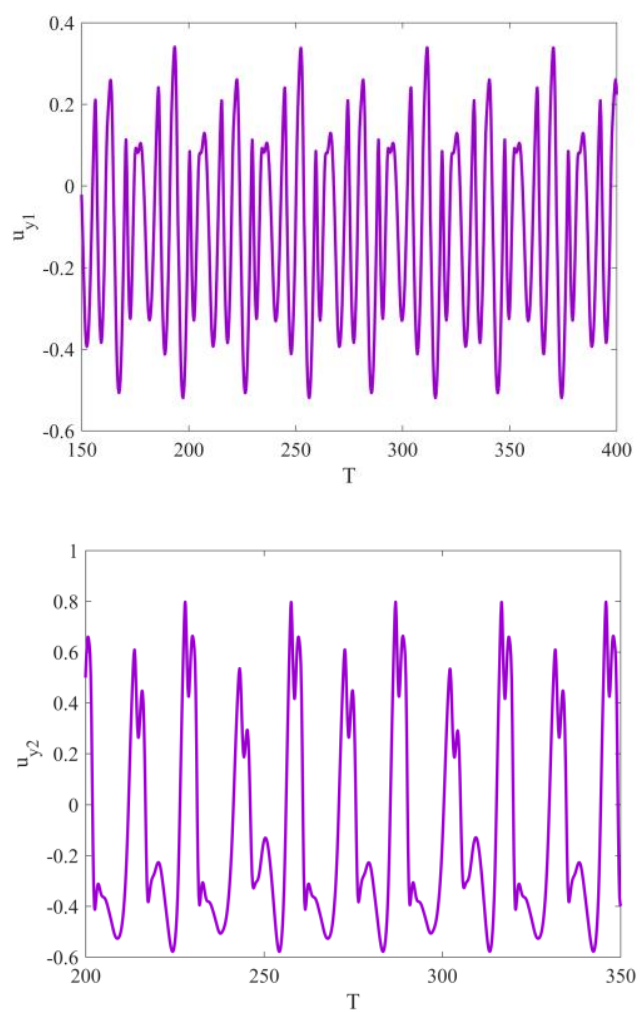

(d)

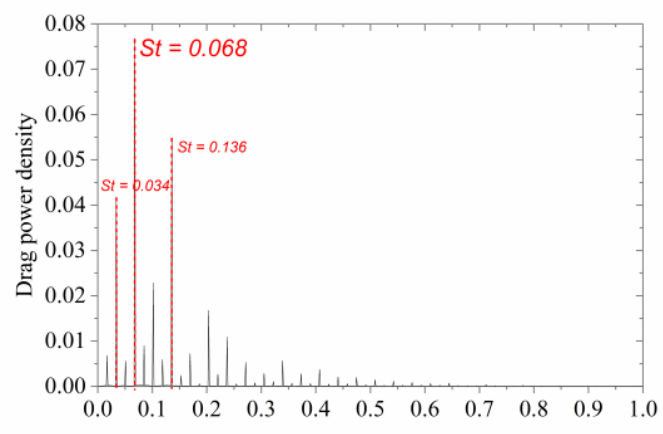

$\mathrm{St}$

(c)

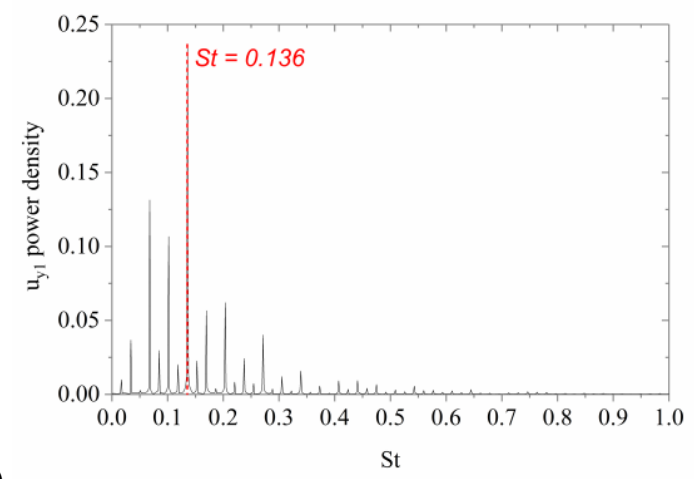

(e)

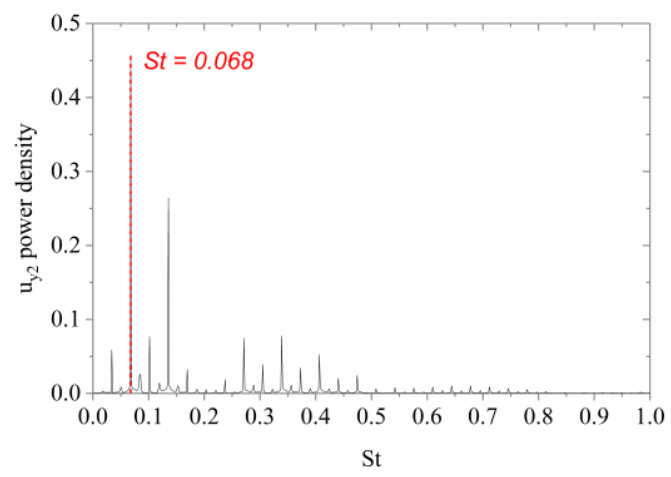

8

(b)

(f) 
1

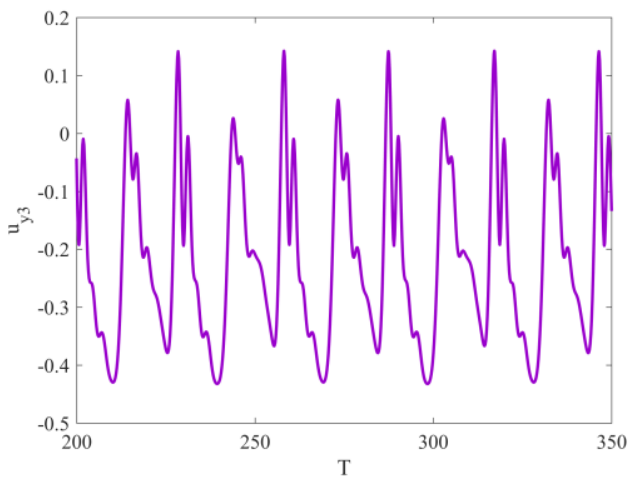

(c)

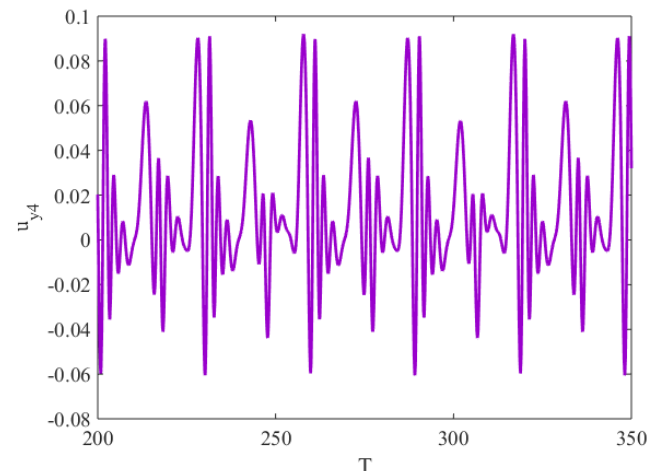

(g)
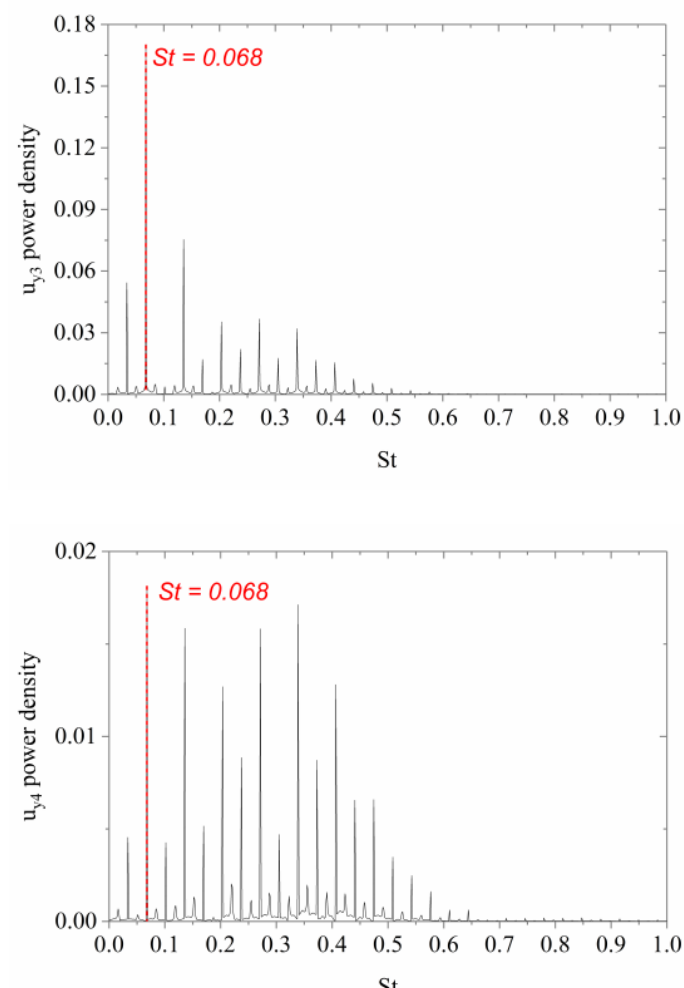

(h)

Figure 17. Y-direction velocity of four points (left) and their spectra (right) with $\alpha=45^{\circ}$ (Indexes 1-4 represent points at (7.5D, 12D), (7D, 10.2D), (6.2D, 9.8D) and (6.2D, 9.3D), respectively.)

6

8

9

10

11

12
5

(d)

\section{(7)}

3.2.5 The flow past two staggered cylinders with $\alpha=46^{\circ}$

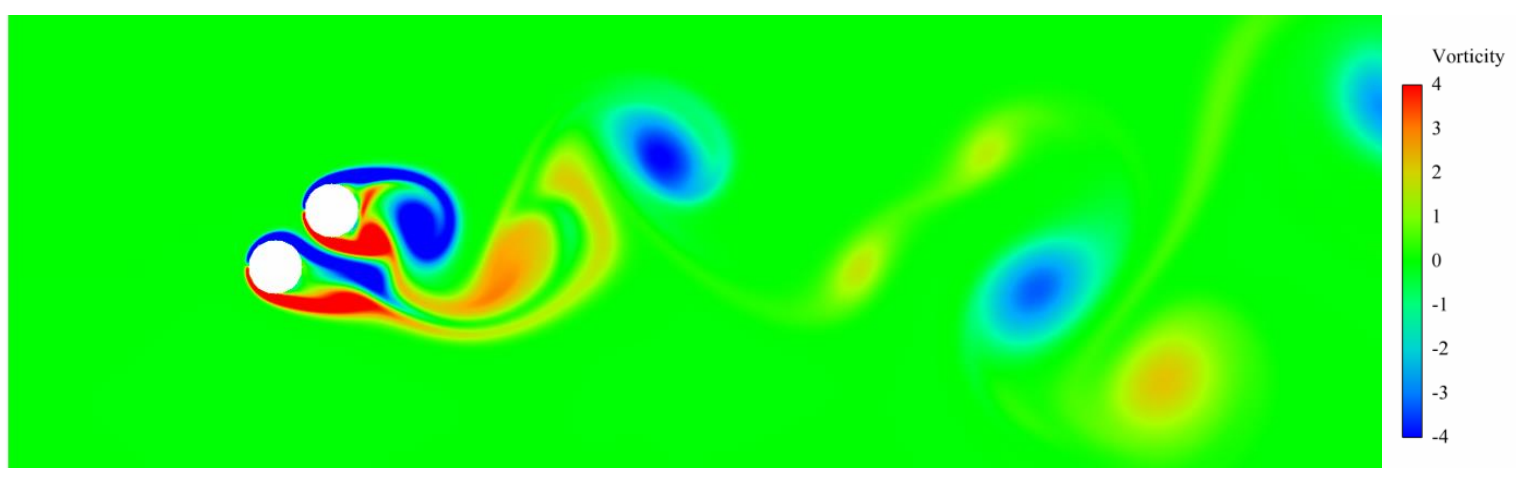

Figure 18. Vortex contour at $\mathrm{T}=217$ with $\alpha=45^{\circ}$

1 Lift and drag coefficients on both cylinders at $\alpha=46^{\circ}$ are shown in Fig. 19. The mean lift and drag coefficients for both cylinders are similar to those in the $\alpha=45^{\circ}$ case. However, what is significant here is that although there is only one-degree change in $\alpha$ from the previous case, the lift and drag coefficients for both cylinders are no longer periodic, which suggests that the oscillation of the flow may become unstable. 
2 We can see from the case of $\alpha=45^{\circ}$, the period of the oscillation becomes very long, 3 although the force history can be still decomposed into Fourier series with discrete frequency 4 components. It could be expected that when the period further increases, the Fourier series 5 will become Fourier transform and the discrete frequency components will become a 6 continuous distribution. This is reflected by the spectra of force coefficients in Fig. 20. The 7 dominant frequency of the lift coefficient for the upper cylinder is $S t=0.133$, and that for the 8 lower cylinder is $S t=0.066$. These two dominant frequencies are reversed for the drag 9 coefficients of two cylinders. The local peaks at $\alpha=46^{\circ}$ resemble those at $\alpha=45^{\circ}$. 10 However, the major difference is that the spectrum at $\alpha=46^{\circ}$ is highly oscillatory between the local peaks, while between two neighbour peaks the amplitudes in the spectrum are virtually zero at $\alpha=45^{\circ}$. Fig.21 shows vortex contour. The vortices are shed from each individual cylinder and then the vortices shed from different cylinders emerge together to form a single wake, which is similar to that in the $\alpha=45^{\circ}$ case. However, based on the detailed analysis the vortex contour over a long period of time it has been found that the oscillatory flow here is no longer periodic and becomes unstable. Thus, unlike that at $\alpha=45^{\circ}$, the Fourier series in time becomes the Fourier transform here.

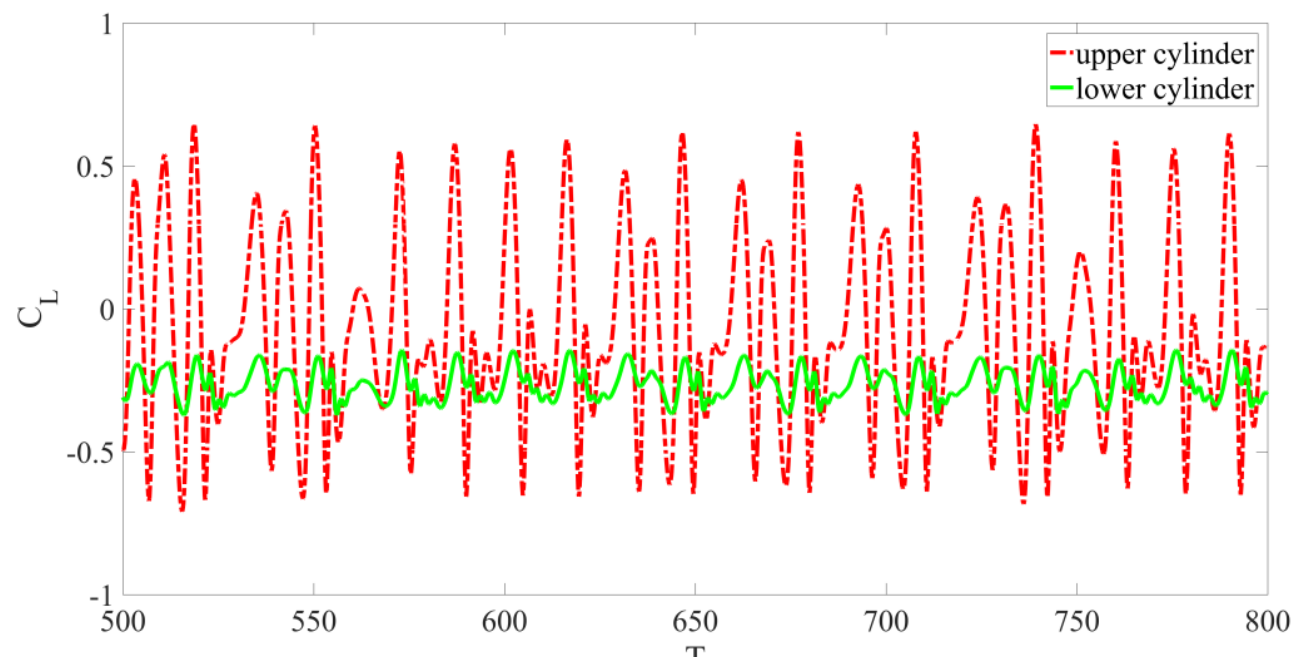

) 


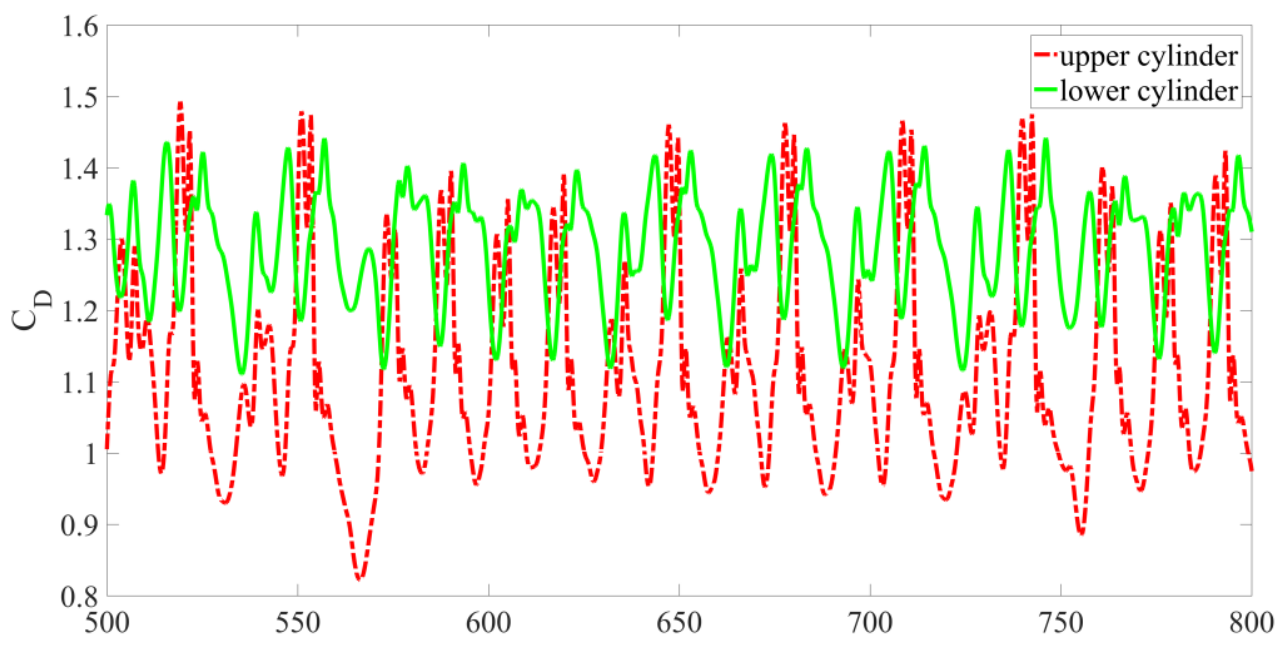

1 (b)

Figure 19. Force coefficient time histories on two cylinders with $\alpha=46^{\circ}$

3

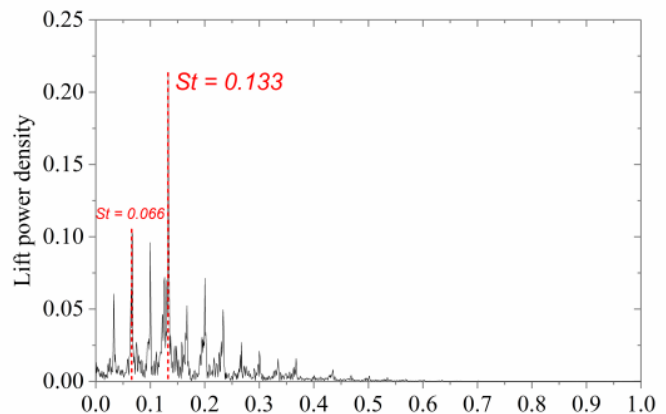

(a)

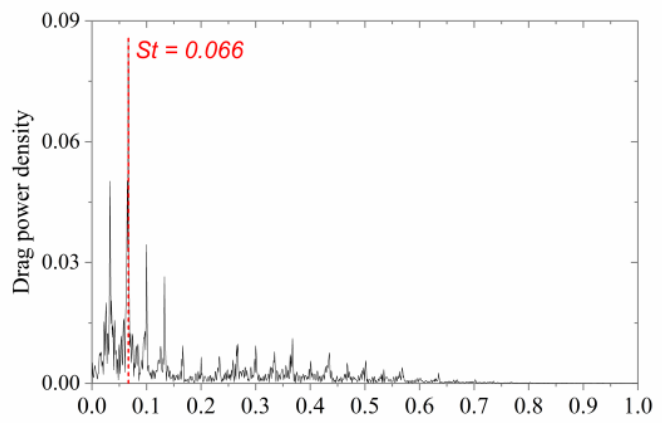

(b)

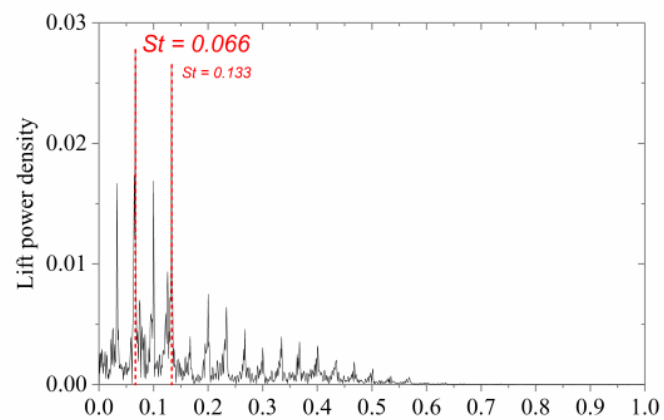

(c)
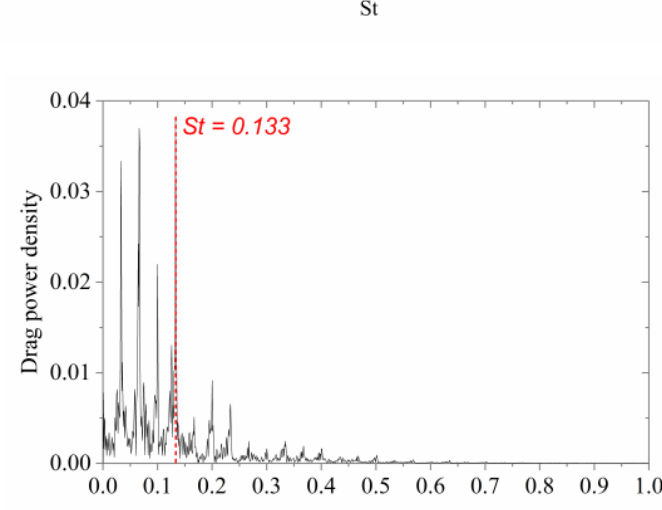

(d)

6 Figure 20. Spectra of force coefficients for (a)-(b) upper cylinder and (c)-(d) lower cylinder with $\alpha=46^{\circ}$ 
3

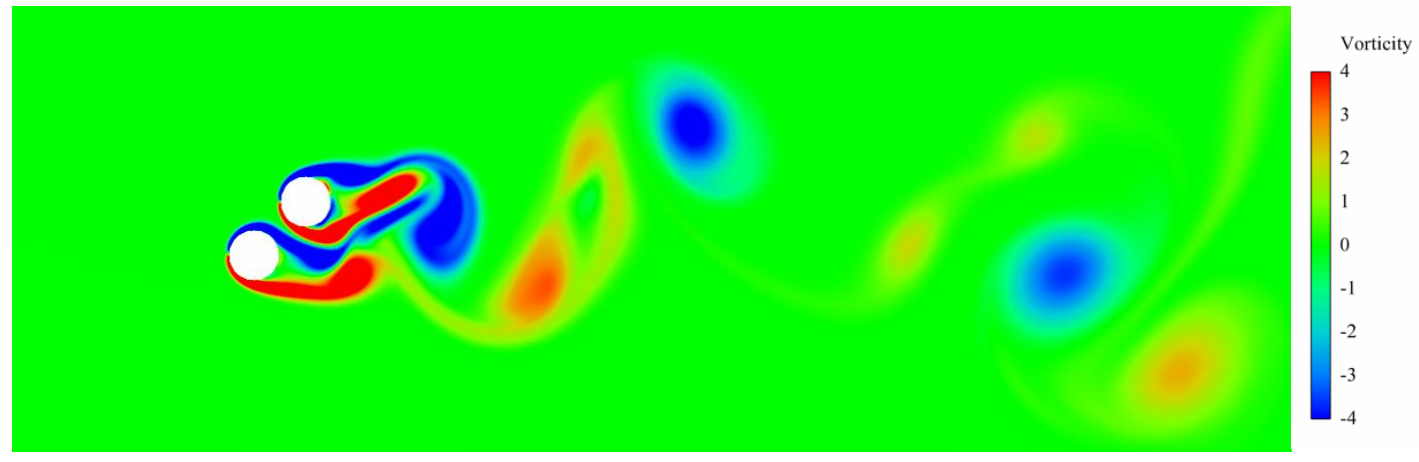

Figure 21. Vortex contour at $\mathrm{T}=637$ with $\alpha=46^{\circ}$

\subsubsection{The flow past two staggered cylinders with $\alpha=60^{\circ}$}

Lift and drag coefficients on both cylinders at $\alpha=60^{\circ}$ are shown in Fig. 22. As in cases of $\alpha=45^{\circ}$ and $\alpha=46^{\circ}$, the mean lift coefficient for the lower cylinder is negative. However, for the upper cylinder, the mean lift coefficient becomes positive, which is opposite to that at $\alpha=45^{\circ}$ and $\alpha=46^{\circ}$. From the analysis of the drag coefficients for two cylinders, it can be found that the mean value for the lower cylinder is higher than that for a single cylinder while for the upper cylinder, it is close to that for a single cylinder.

Frequency spectra of the force coefficients on two cylinders are given in Fig. 23. As in cases of $\alpha=45^{\circ}$ and $\alpha=46^{\circ}$, the locations of spectrum peaks of the lift coefficients for upper and lower cylinders are different, and are at $S t=0.107$ and $S t=0.08$, respectively. The peaks for drag forces on two cylinders are both at $S t=0.08$. It is interesting to see that the amplitude of the drag coefficient at a much smaller frequency $S t=0.027$ is also high and it is close to that at the dominant frequency. The importance of much smaller frequency may reflect the slow change of the gap flow bias. It is obvious that the flow past each of the two cylinders at $\alpha=60^{\circ}$ takes irregular turns in experiencing narrower or wider wake patterns in Fig. 24 or flip-flopping occurs. Moreover, three frequencies for Fourier analyses on the drag coefficients may be distinguished: a low flip-flopping frequency $S t=0.027$, two dominant frequencies $S t=0.08$ and $S t=0.107$. It is worthwhile to notice that the low flip-flopping frequency $S t=0.027$ plus the vortex-shedding frequency $S t=0.08$ equals the third distinctive component $S t=0.107$, which was also discussed in Carini et al. (2014). 


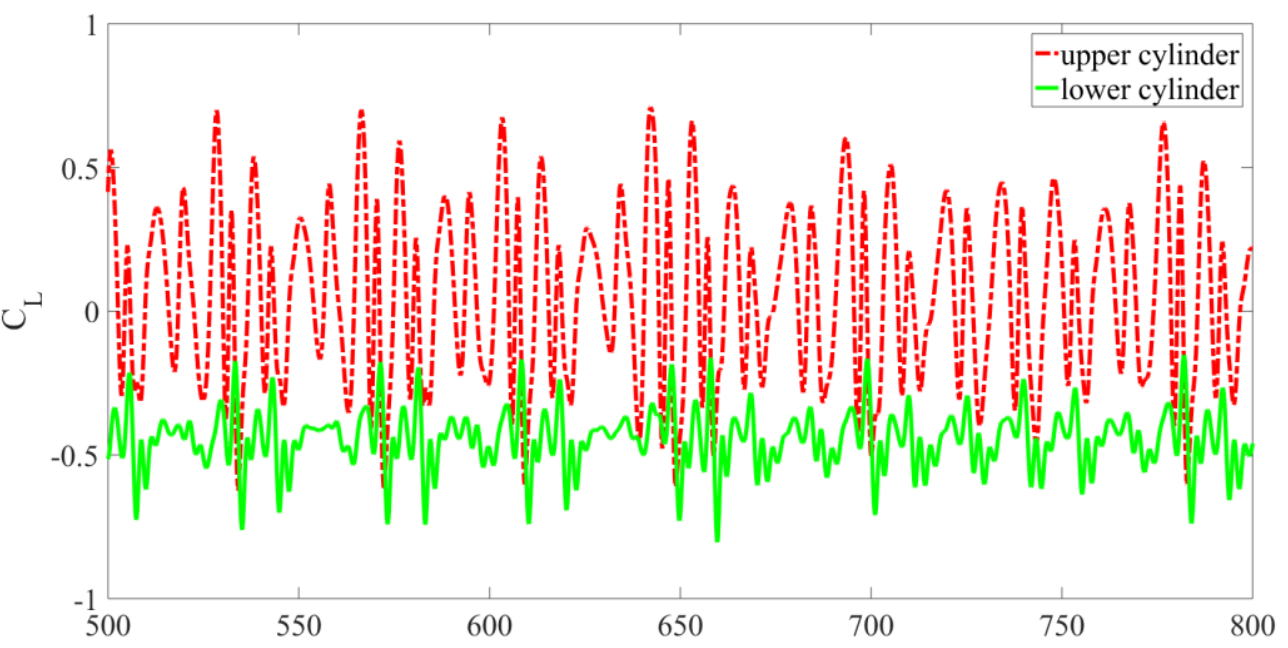

$1 \quad$ (a)

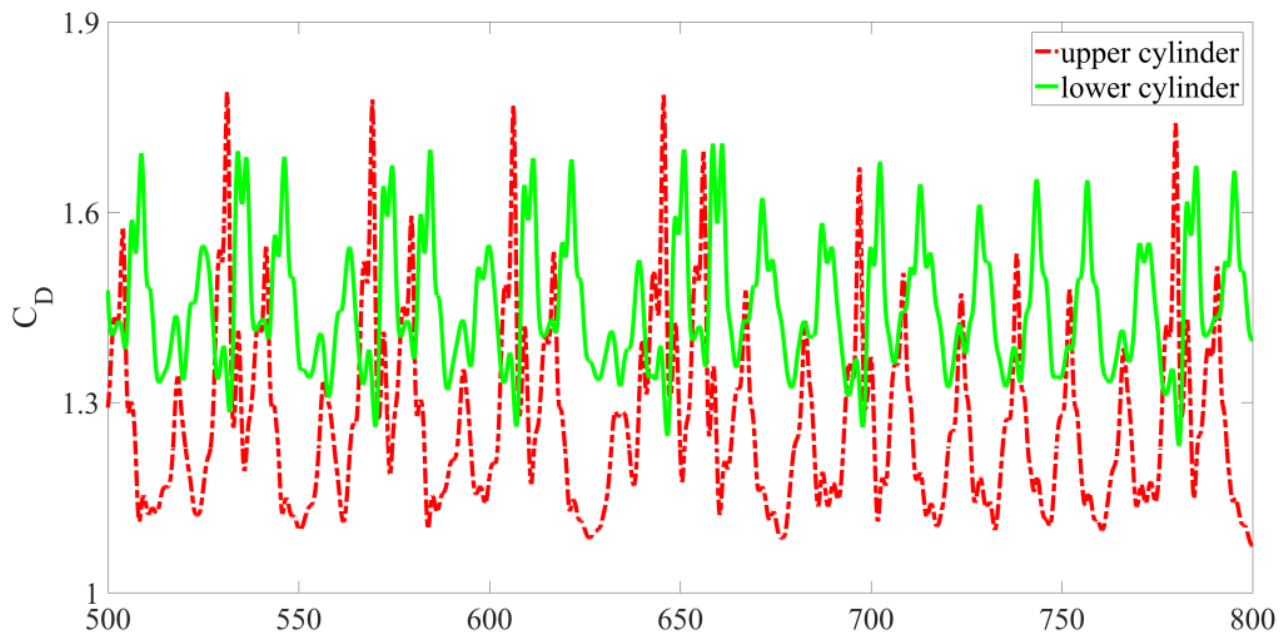

(b)

Figure 22. Force coefficient time histories on two cylinders with $\alpha=60$

4

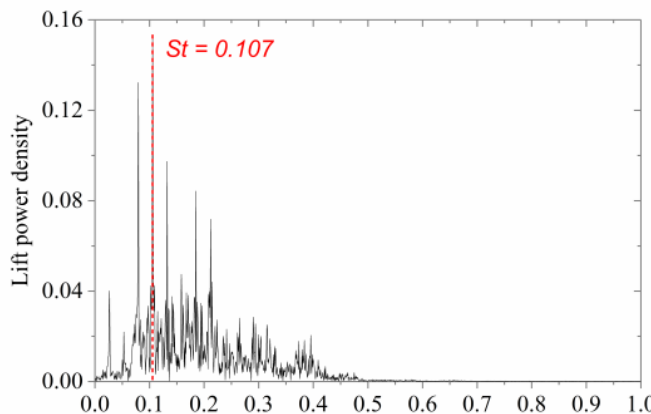

(a)

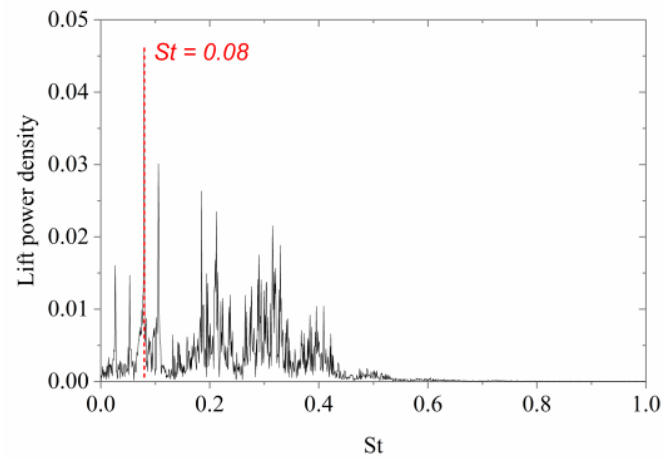

(c) 


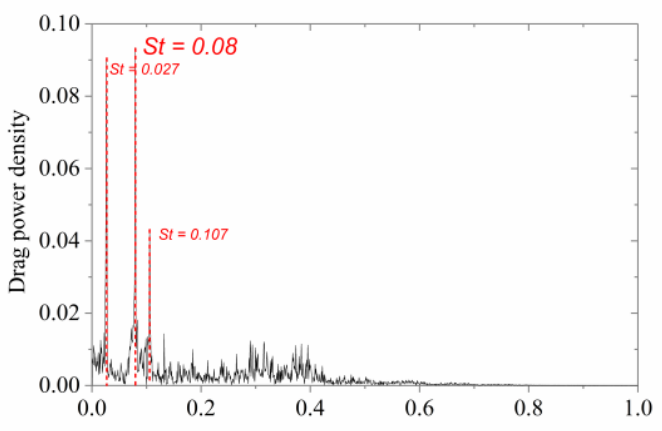

(b)

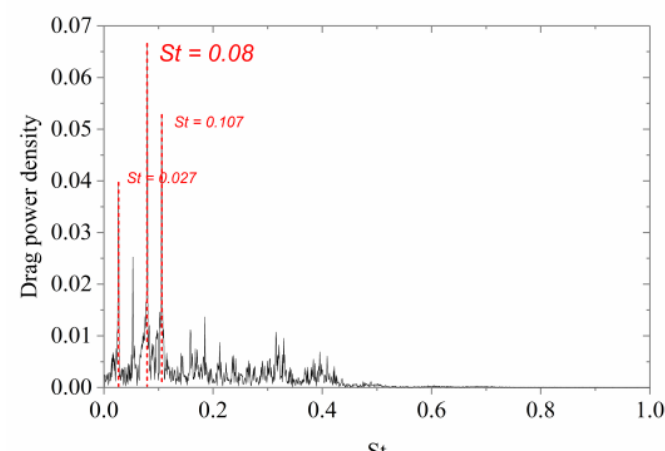

(d)

2 Figure 23. Spectra of force coefficients for (a)-(b) upper cylinder and (c)-(d) lower cylinder with $\alpha=60^{\circ}$

(a)

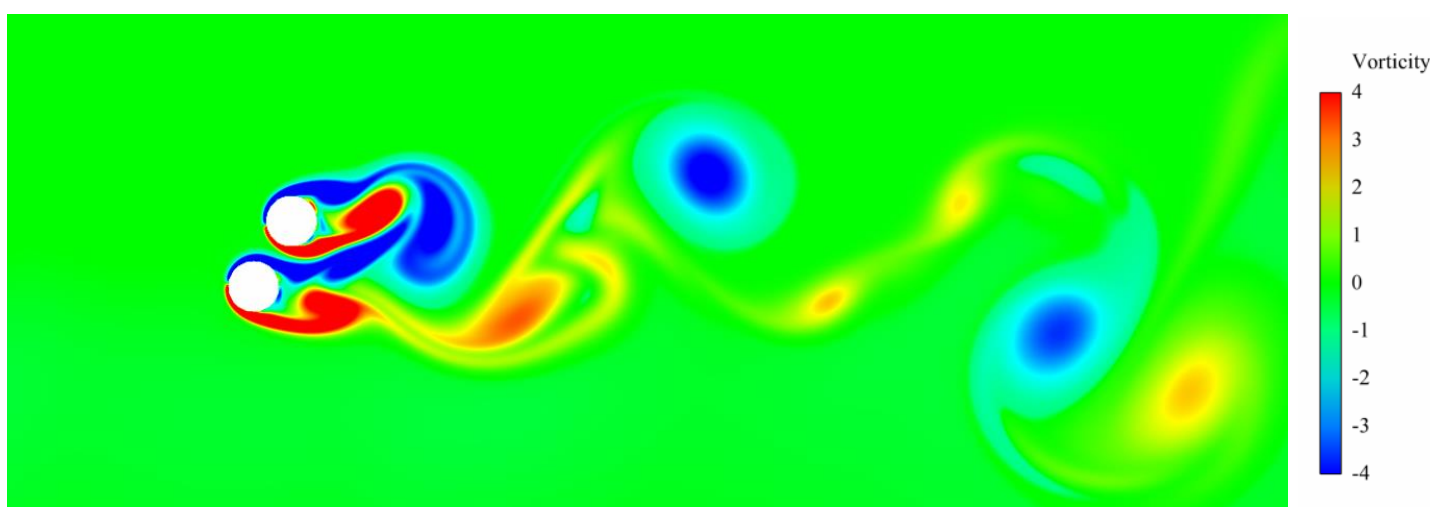

5

10 Lift and drag coefficients on both cylinders at $\alpha=90^{\circ}$ are shown in Fig. 25. As the 11 configuration is once again symmetric, the mean drag coefficients for two cylinders become 12 similar. Analysis of the data has shown that the mean values for both cylinders are higher 13 than that for a single cylinder. The mean lift coefficient is positive for the upper cylinder and 14 negative for the lower cylinder, and thus there is a repulsive mean force between two 
1 cylinders. Furthermore, $C_{D}$ and $C_{L}$ on both cylinders yield irregular temporal variations 2 around the mean value.

4 Spectra of force on each individual cylinder are given in Fig. 26. At $\alpha=90^{\circ}$, the flow 5 oscillation is at the random flip-flopping state. In fact, this depends on how the centre-tocentre spacing ratio $L^{*}$ is deliberately chosen, as discussed in the introduction. In this flow, 7 the narrow and wide wakes behind each cylinder alternate irregularly. Fig. 27 shows two snapshots as an example. Even though oscillatory forces here are very different between the upper and lower cylinders, their frequency spectra for two cylinders are similar. There are multiple intricate frequencies and a large number of relatively high peaks in spectra. The dominant frequencies for the lift coefficient on each individual cylinder are no longer a single value. They are scattered over a relatively broad range, at approximately $0.13 \leq S t \leq 0.22$. The upper limit of this range is similar to the dominant frequency for a single cylinder. The reason for this range to be chosen is that outside this range the peaks in frequency spectra are much smaller. The frequency $S t=0.057$ corresponding to the spectrum peak of drag forces for the two cylinders is much smaller. Two ranges in the frequency spectra can be seen: a lower flip-flopping frequency range at $S t<0.1$ and a higher oscillatory frequency range $0.1<S t<0.5$. Together with previous two-cylinder cases, it can be found that with the increase of alignment angle $\alpha$, the oscillatory forces are no longer dominated by a component at a single frequency. As $\alpha$ further increases, frequency spectra of lift and drag coefficients change from the discrete components to the continuous distribution and the dominant frequency components in their spectra become more complex.

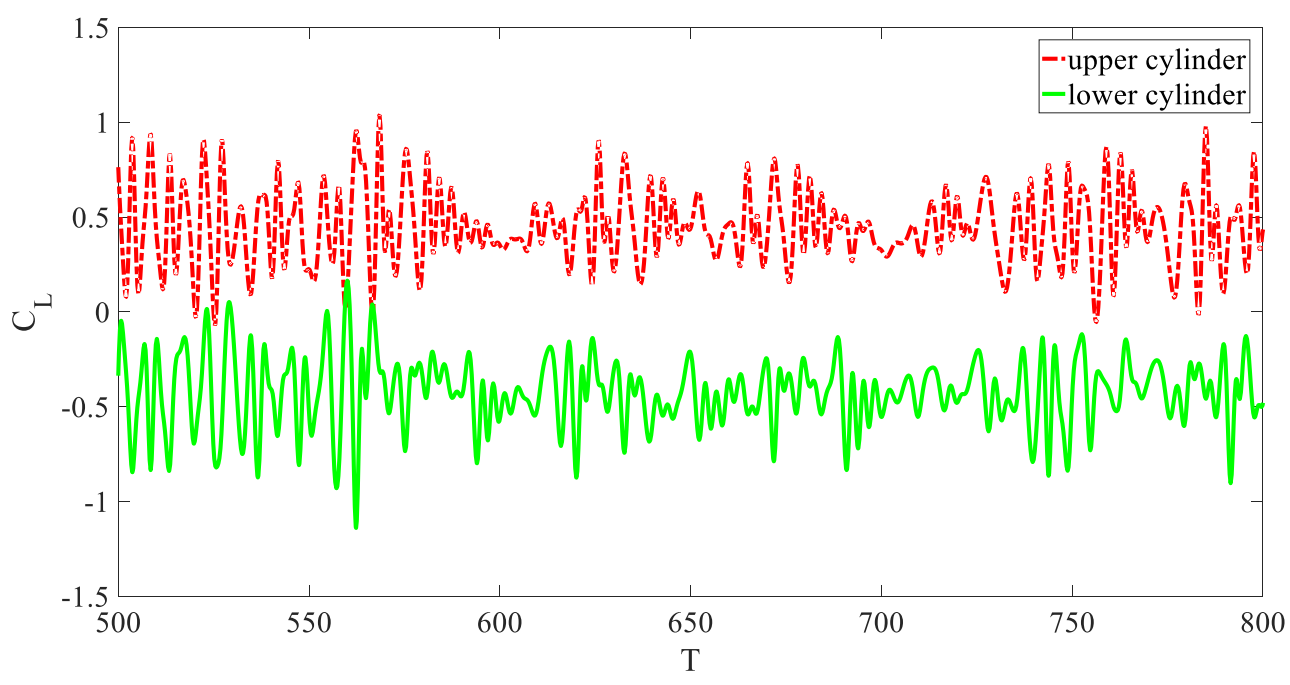


1

(b)

2

3

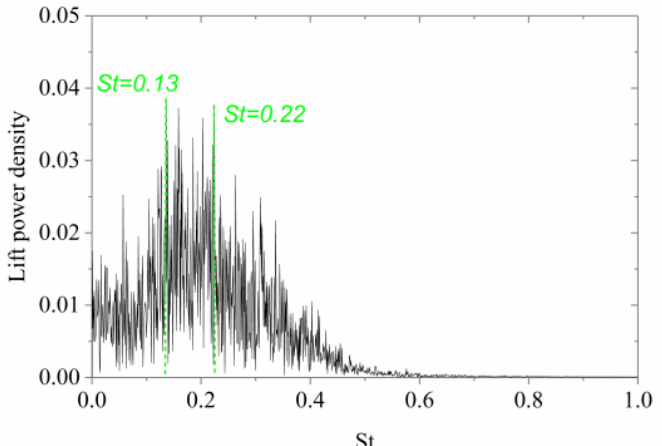

(a)

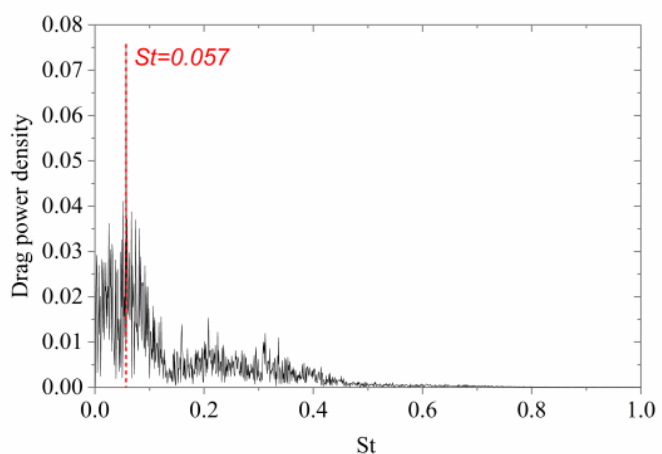

(b)

(d)

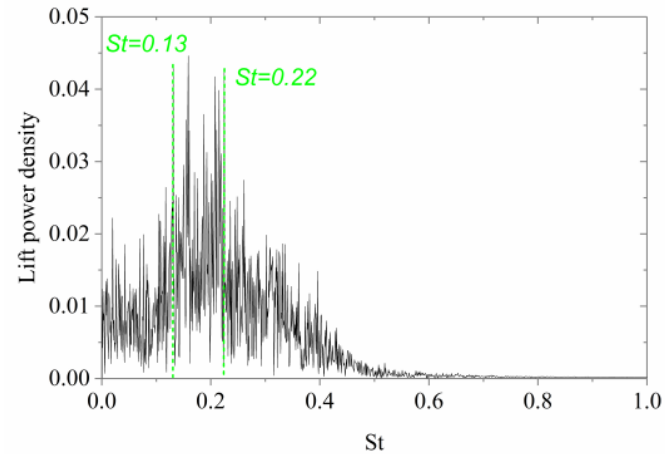

(c)

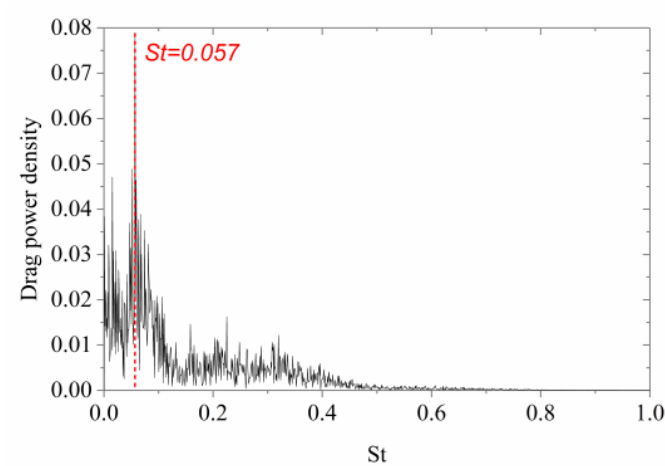

6 Figure 26. Spectra of force coefficients for (a)-(b) upper cylinder and (c)-(d) lower cylinder with $\alpha=90^{\circ}$ 
(a)
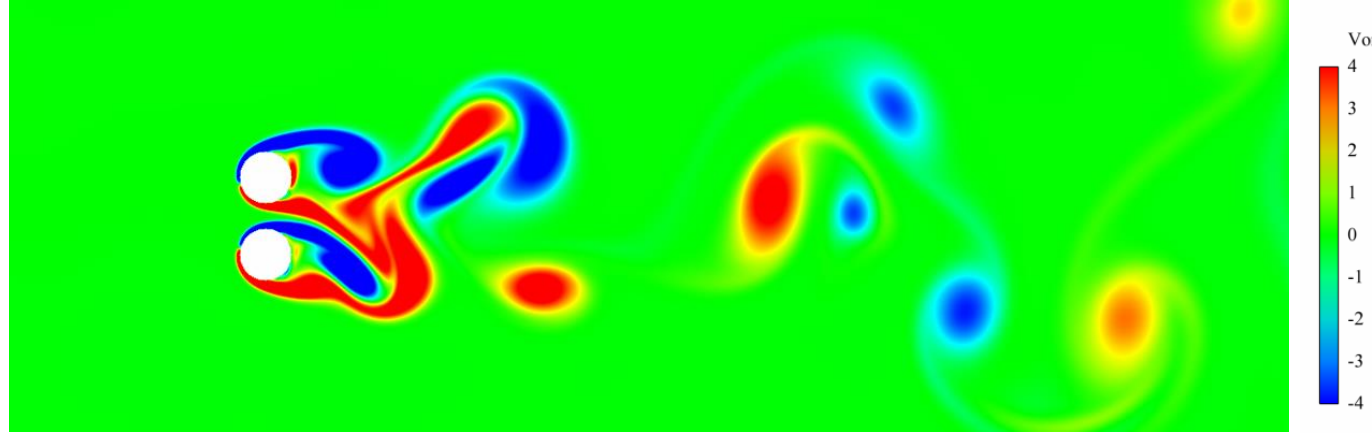

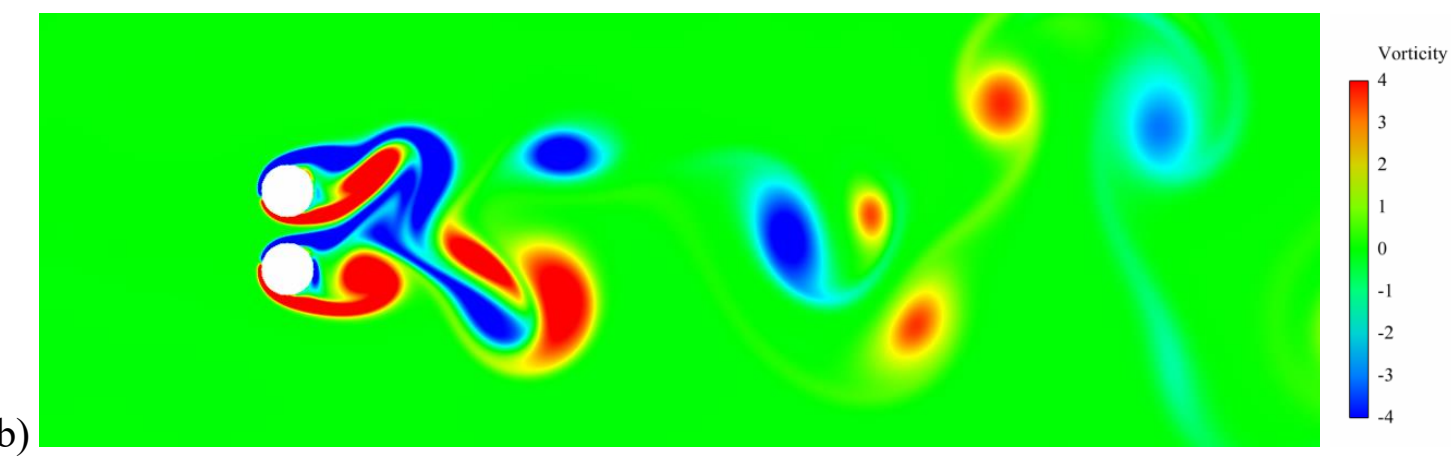

Figure 27. Vortex contour with $\alpha=90^{\circ}$ at (a) $\mathrm{T}=693$ and (b) $\mathrm{T}=721$

\section{Conclusions}

Flow past two cylinders in different arrangements has been investigated through numerical simulations based on lattice Boltzmann method together with immersed boundary method. The focus has been on how the force on each cylinder varies with alignment angle $\alpha$, as well as the vortex contour. Simulations have been performed for two cylinders with the intermediate spacing $L^{*}=1.5$ at the Reynolds number $\operatorname{Re}=200$ and at different $\alpha$. From the results, the following conclusions can be drawn.

4 When $0^{\circ} \leq \alpha \leq 45^{\circ}$, flow is always periodic with period $T_{v}$, which gives a lowest frequency $5 f_{v}=1 / T_{v}$. The force is also periodic. However, there could be frequency components $n f_{v}$ $16 \quad(n=1,2,3 \cdots)$. At $\alpha=0^{\circ}$, the lift coefficients $C_{L}$ on both cylinders have components of $7(2 n+1) f_{v}$ and drag coefficients $C_{D}$ have $2 n f_{v}$, although only first couple of components are 8 visible. This is similar to that for a single cylinder. When $\alpha$ is non zero, the flow 9 configuration becomes asymmetric and both $C_{L}$ and $C_{D}$ have components $n f_{v}$. Moreover, 20 as increases, the number of frequency components, at which the force is significant, also 21 increases. While the lowest frequency becomes smaller, these frequency components become 
1 wider spread. As a result, the force oscillation becomes less sinusoidal even though it remains periodic. For $\alpha \geq 30^{\circ}$, the lowest frequency is no longer the dominant component. At $\alpha=45^{\circ}$,

3 the dominant frequency of the lift force on the upper cylinder is not the same as that of the other three forces. This is very much due to the oscillation of the upper shear layer of the upper cylinder, which has a dominant frequency different from that corresponding to other shear layers. When $\alpha>45^{\circ}$, the motion is no longer periodic and becomes unstable. The force spectrum changes from the discrete frequency distribution to a continuous distribution, or the Fourier series becomes the Fourier transform. When $\alpha$ further increases, flip-flopping state with low frequency may start to develop. At $\alpha=60^{\circ}$, three distinctive frequencies of force coefficients have been observed. The lowest is the flip-flopping frequency and the second one is the dominant frequency due to wake oscillation, while the summation of these two equals to the third component. At $\alpha=90^{\circ}$, a large number of relatively high peaks have been seen in the force spectrum and the dominant force is over a relatively broad range of frequencies. Interestingly, although two cylinders experience irregular force oscillation at $\alpha=90^{\circ}$, their frequency spectra are similar to each other.

\section{Acknowledgements}

The first author is sponsored by the scholarship of China Scholarships Council, to which she is most grateful. The authors also gratefully acknowledge financial support from the Lloyd's Register Foundation (LRF) through the joint centre involving University College London, Shanghai Jiao Tong University and Harbin Engineering University. The LRF helps to protect life and property by supporting engineering-related education, public engagement and the application of research.

\section{References}

Aidun, C.K., Clausen, J.R., 2010. Lattice-Boltzmann method for complex flows. Annual Review of Fluid Mechanics 42, 439-472.

Akbari, M.H., Price. S.J., 2005. Numerical investigation of flow patterns for staggered cylinder pairs in crossflow. Journal of Fluids and Structures 20, 533-554.

Bearman, P.W., Wadcock, A.J., 1973. The interaction between a pair of circular cylinders normal to a stream. 
Journal of Fluid Mechanics 61, 499-511.

2

3 Braza, M., Chassaing, P., Ha Minh, H. 1986. Numerical study and physical analysis of the pressure and velocity

4 fields in the near wake of a circular cylinder. Journal of Fluid Mechanics 165, 79-130.

5

6 Carini, M., Giannetti, F., Auteri, F., 2014. On the origin of the flip-flop instability of two side-by-side cylinder

7 wakes. Journal of Fluid Mechanics 742, 552-576.

8

9 Carmo, B.S., Meneghini, J.R., 2006. Numerical investigation of the flow around two circular cylinders in 10 tandem. Journal of Fluids and Structures 22, 979-988.

11

Cercignani, C., 1988. The Boltzmann Equation and Its Application. Spring, New York.

13

14 Chan, C.T., Anastasiou, K., 1999. Solution of incompressible flows with or without a free surface using the 15 finite volume method on unstructured triangular meshes. International Journal on Numerical Methods in Fluids 16 $29,35-57$.

17

18 Chen, S., Doolen, G. 1998. Lattice Boltzmann method for fluid flows. Annual Review of Fluid Mechanics 30, $19329-364$.

20

Chen, Y.H., Yang, S.C., Yang, J.Y., 1999. Implicit weighted essentially non-oscillatory schemes for the incompressible Navier-Stokes equations. International Journal on Numerical Methods in Fluids 31,747-765.

23

Hu, J., Zhou, Y., 2008a. Flow structure behind two staggered circular cylinders. Part 1. Downstream evolution and classification. Journal of Fluid Mechanics 607, 51-80.

Hu, J., Zhou, Y., 2008b. Flow structure behind two staggered circular cylinders. Part 2. Heat and momentum transport. Journal of Fluid Mechanics 607, 81-107.

Igarashi, T., 1981. Characteristics of the flow around two circular cylinders arranged in tandem ( $1^{\text {st }}$ report). Bulletin of the JSME 24, 323-331.

Jester, W., Kallinderis, Y., 2003. Numerical study of incompressible flow about fixed cylinder pairs. Journal of Fluids and Structures 17, 561-577.

Kang, S., 2003. Characteristics of flow over two circular cylinders in a side-by-side arrangement at low Reynolds numbers. Physics of Fluids 15, 2486-2497.

Kim, H.J., Durbin, P.A., 1988. Investigation of the flow between a pair of circular cylinders in the flopping regime. Journal of Fluid Mechanics 196, 431-448. 
arrangement. ASME Journal of Fluids Engineering 102, 166-173.

2

3 Lai, M.C., Peskin, C.S., 2000. An immersed boundary method with formal second-order accuracy and reduced

4 numerical viscosity. Journal of Computational Physics 160, 705-719.

5

6 Lima, L.F., Silva, E., Silveira-Neto, A., Damasceno, J.J.R., 2003. Numberical simulation of two-dimensional

7 flows over a circular cylinder using the immersed boundary method. Journal of Computational Physics 189,

$8 \quad 351-370$.

9

10 Li, Y., Shoch, R., Zhang, R., Chen, H., 2004. Numerical study of flow past an impulsively started cylinder by 11 the lattice-Boltzmann method. Journal of Fluid Mechanics 519, 273-300.

13 Li, Z., Favier, J., D’Ortona, U., Poncet, S., 2016. An immersed boundary-lattice Boltzmann method for single14 and multi-component fluid flows. Journal of Computational Physics 304, 424-440.

Meneghini, J.R., Saltara, F., Siqueira, C.L.R., Ferrari Jr., J.A., 2001. Numerical simulation of flow interference between two circular cylinders in tandem and side-by-side arrangements. Journal of Fluids and Structures 15, 327-350.

19

Mittal, S., Kumar, V., Raghuvanshi, A., 1997. Unsteady incompressible flows past two cylinders in tandem and staggered arrangements. International Journal for Numerical Methods in Fluids 25, 1315-1344.

22

Peng, Y., Shu, C., Chew, Y.T., Niu, X.D., Lu, X.Y., 2006. Application of multi-block approach in the immersed boundary-lattice Boltzmann method for viscous fluid flows. Journal of Computational Physics 218, 460-478.

Peskin, C.S., 1977. Numerical analysis of blood flow in the heart. Journal of Computational Physics 25, 220252.

Peskin, C.S., 2002. The immersed boundary method. Acta Numerica 11, 479-517.

Qian, Y.H., d'Humieres D., Lallemand P., 1992. Lattice BGK models for Navier-Stokes equation. Europhysics Letters 17(6), 479-484.

Singha, S., Nagarajan, K.K., Sinhamahapatra, K.P., 2016. Numerical study of two-dimensional flow around two side-by-side ciecular cylinders at low Reynolds numbers. Physics of Fluids 28, 053603. 
Sumner, D., 2010. Two circular cylinders in cross-flow: A review. Journal of Fluids and Structures 26, 849-899.

2

3 Sumner, D., Price, S.J., Paidoussis, M.P., 2000. Flow-pattern identification for two staggered circular cylinders

4 in cross-flow. Journal of Fluid Mechanics 411, 263-303.

5

6 Sumner, D., Wong, S.S.T., Price, S.J., Paidoussis, M.P., 1999. Fluid behaviour of side-by-side circular cylinders

7 in steady cross-flow. Journal of Fluids and Structures 13, 309-338.

8

9 Tong, F., Liang, C., Zhao, M., 2015. Numerical simulations of steady flow past two cylinders in staggered 10 arrangements. Journal of Fluid Mechanics 765, 114-149.

11

12 Williamson, C.H.K., 1996. Vortex dynamics in the cylinder wake. Annual Review of Fluid Mechanics 28, 47713539.

14

15 Williamson, C.H.K., 1985. Evolution of a single wake behind a pair of bluff bodies. Journal of Fluid Mechanics $16 \quad 159,1-18$.

17

18 Wolf-Gladrow, D.A., 2000. Lattice-gas Cellular Automata and Lattice Boltzmann Medols: An Introduction. New 19 York: Springer.

20

Wu, G.X., Hu, Z.Z. 2006. Numerical simulation of viscous flow around unrestrained cylinders. Journal of Fluid Structures 22, 371-390.

23

24 Wu, G.X., 2000. A note on non-linear hydrodynamics force on a floating body. Applied Ocean Research 22, $25 \quad 315-316$.

Wu, J., Shu, C, 2009. Implicit velocity correction-based immersed boundary-lattice Boltzmann method and its applications. Journal of Computational Physics 228, 1963-1979. 272.

Zdravkovich, M.M., 2003. Flow Around Circular Cylinder, vol. 2: Applications. Oxford University Press, 33 Oxford, UK.

Zdravkovich, M.M., 1987. The effects of interference between circular cylinders in cross flow. Journal of Fluids and Structures 1, 239-261.

38 Zdravkovich, M.M., 1977. Review of flow interference between two circular cylinders in various arrangements. 
1 Zhou, Y., Zhang, H.J., Yiu, M.W., 2002. The turbulent wake of two side-by-side circular cylinders. Journal of 2 Fluid Mechanics 458, 303-332. 